\title{
Sur les automorphismes du groupe de Cremona
}

\author{
Julie Déserti
}

\begin{abstract}
We prove that an automorphism of the group of birational transformations of the complex projective plane is the composition of an interior automorphism and an automorphism of the field $\mathbb{C}$. The proof is based on a study of maximal abelian subgroups of the Cremona group.
\end{abstract}

\section{Introduction}

L'étude des automorphismes des groupes a fait l'objet de nombreux travaux ; dans le cas des groupes 'classiques' on pourra consulter le livre de Dieudonné [Die55]. Par exemple, le groupe d'automorphismes de l'espace projectif $\mathbb{P}^{n}(\mathbb{C})$ coïncide avec $\operatorname{PGL}(n+1, \mathbb{C}) ;$ les automorphismes de ce groupe sont engendrés par les automorphismes intérieurs, la contragrédiente $u \mapsto{ }^{t} u^{-1}$ et les automorphismes du corps $\mathbb{C}$. La preuve présentée par Dieudonné repose sur le fait que tout automorphisme de $\operatorname{PGL}(n+1, \mathbb{C})$ provient par passage au quotient d'un automorphisme de $\mathrm{GL}(n+1, \mathbb{C})$ et sur l'étude de certains éléments de $\mathrm{GL}(n+1, \mathbb{C})$ d'ordre deux, les 'involutions extrémales'. Ce résultat peut être retrouvé en étudiant les sous-groupes abéliens et résolubles maximaux de $\operatorname{GL}(n+1, \mathbb{C})$.

En 1963, Whittaker montre que tout isomorphisme entre les groupes d'homéomorphismes de deux variétés topologiques connexes est induit par un homéomorphisme entre les variétés ellesmêmes [Whi63]. En 1982, Filipkiewicz propose un résultat semblable pour les variétés différentiables [Fil82] ; si $M$ est une variété de classe $\mathcal{C}^{k}$, notons $\operatorname{Diff}^{k}(M)$ le groupe des difféomorphismes de classe $\mathcal{C}^{k}$ sur $M$.

ThÉORÈme 1.1 [Fil82]. Soient $M$ et $N$ deux variétés connexes respectivement de classe $\mathcal{C}^{k}$ et $\mathcal{C}^{j}$. Si $\varphi: \operatorname{Diff}^{k}(M) \rightarrow \operatorname{Diff}^{j}(N)$ est un isomorphisme de groupes, alors $k$ est égal à $j$ et il existe un difféomorphisme $\psi: M \rightarrow N$ de classe $\mathcal{C}^{k}$ tel que, pour tout $f$ dans $\operatorname{Diff}^{k}(M)$, on ait :

$$
\varphi(f)=\psi f \psi^{-1} \text {. }
$$

Filipkiewicz reprend l'idée de Whittaker : il montre qu'un isomorphisme de groupes $\varphi$ induit une bijection $\psi$ entre les stabilisateurs de points de $M$ et ceux de $N$. D'après un résultat de Takens [Tak79], si $j=k=\infty$, alors $\psi$ est un $\mathcal{C}^{\infty}$-difféomorphisme. Dans le cas général, Filipkiewicz utilise un résultat de Mongtgomery et Zippin [MZ55] sur les groupes de Lie agissant par difféomorphismes sur les variétés pour montrer que $k$ et $j$ sont égaux et que $\psi$ est un $\mathcal{C}^{k}$ difféomorphisme. Nous renvoyons à [Ban86] et [Ban97] pour des résultats analogues dans différents contextes (groupe des difféomorphismes qui préservent une forme volume, une forme de contact ou une forme symplectique).

Soit $V$ une variété algébrique complexe définie sur $\mathbb{Q}$; notons $\operatorname{Aut}(V)$ le groupe des biholomorphismes de $V$ et $\operatorname{Bir}(V)$ le groupe des transformations birationnelles de $V$. Puisque $V$ est définie

Received 22 January 2006, accepted in final form 16 June 2006.

2000 Mathematics Subject Classification 14E07 (primary), 14E05 (secondary).

Keywords: birational transformations, fibration, automorphism.

This journal is (C) Foundation Compositio Mathematica 2006. 


\section{J. DÉSERTI}

sur $\mathbb{Q}$, un automorphisme $\tau$ du corps $\mathbb{C}$ induit un isomorphisme $\tau($.$) de \operatorname{Aut}(V)$ (respectivement $\operatorname{Bir}(V))$ : par exemple lorsque $V=\mathbb{P}^{n}(\mathbb{C})$, à un élément $f$ de $\operatorname{Bir}(V)$ nous associons l'élément $\tau(f)$ obtenu en faisant agir $\tau$ sur les coefficients de $f$ exprimé en coordonnées homogènes. Dans [Dés06] nous établissons un analogue du théorème 1.1 pour le groupe Aut $\left[\mathbb{C}^{2}\right]$ des automorphismes polynomiaux du plan $\mathbb{C}^{2}$ : un automorphisme du groupe Aut $\left[\mathbb{C}^{2}\right]$ est la composée d'un automorphisme intérieur et de l'action induite par un automorphisme du corps $\mathbb{C}$ (ce résultat est en fait valable pour tout corps non dénombrable de caractéristique nulle, voir [Dés06]). Le but du texte est d'étendre cet énoncé au groupe $\operatorname{Bir}\left(\mathbb{P}^{2}(\mathbb{C})\right)$ des transformations birationnelles du plan projectif complexe, encore appelé groupe de Cremona. Dans une carte affine $(x, y)$, si $f$ est un élément de $\operatorname{Bir}\left(\mathbb{P}^{2}(\mathbb{C})\right.$ ), nous noterons $f$ par ses deux composantes $\left(f_{1}(x, y), f_{2}(x, y)\right)$; par exemple $(1 / x, 1 / y)$ désigne l'involution de Cremona. Rappelons le théorème de Nother, valable pour tout corps algébriquement clos :

ThÉorème 1.2 [Alb02, Cas01]. Le groupe $\operatorname{Bir}\left(\mathbb{P}^{2}(\mathbb{C})\right)$ est engendré par $\operatorname{PGL}(3, \mathbb{C})$ et l'involution de Cremona $\sigma=(1 / x, 1 / y)$.

Le but principal de ce texte est de démontrer le théorème suivant :

ThÉorème 1.3. Soit $\varphi$ un automorphisme du groupe de Cremona. Il existe un automorphisme $\tau$ du corps $\mathbb{C}$ et un élément $\psi$ de $\operatorname{Bir}\left(\mathbb{P}^{2}(\mathbb{C})\right)$ tels que, pour toute transformation birationnelle $f$, nous ayons :

$$
\varphi(f)=\tau\left(\psi f \psi^{-1}\right)
$$

Autrement dit, le groupe des automorphismes extérieurs de $\operatorname{Bir}\left(\mathbb{P}^{2}(\mathbb{C})\right)$ s'identifie au groupe des automorphismes du corps $\mathbb{C}$.

Nous en déduisons, en particulier, le résultat suivant.

Corollaire 1.4. Soient $S$ une surface projective complexe et $\varphi$ un isomorphisme entre $\operatorname{Bir}(S)$ et $\operatorname{Bir}\left(\mathbb{P}^{2}(\mathbb{C})\right)$. Il existe une transformation birationnelle $\psi: S \rightarrow \mathbb{P}^{2}(\mathbb{C})$ et un automorphisme $\tau d u$ corps $\mathbb{C}$ tels que, pour tout $f$ dans $\operatorname{Bir}(S)$, nous ayons :

$$
\varphi(f)=\tau\left(\psi f \psi^{-1}\right) .
$$

Pour l'étude des automorphismes de Aut $\left[\mathbb{C}^{2}\right]$, nous avons largement utilisé les travaux de Lamy (voir [Lam01]) ; la structure de produit amalgamé de ce groupe (théorème de Jung, [Jun42], [Lam02]) induit une action non triviale de Aut $\left[\mathbb{C}^{2}\right]$ sur un arbre, action qui s'avère cruciale dans la démarche de Lamy. Nous ne disposons pas d'un tel objet pour le groupe de Cremona ; la preuve est donc essentiellement différente. Présentons en quelques mots l'idée de notre démonstration. Remarquons que l'image par $\varphi$ d'un sous-groupe abélien maximal du groupe de Cremona est encore abélien maximal ; la compréhension de l'action de $\varphi$ repose donc naturellement sur la description de ce type de groupes.

Notons J le groupe d'invariance de la fibration standard $y=$ cte ; ce groupe, appelé groupe de Jonquières, est isomorphe au produit semi-direct $\operatorname{PGL}(2, \mathbb{C}(y)) \rtimes \operatorname{PGL}(2, \mathbb{C})$.

L'observation suivante (lemme 2.1) s'avère importante : tout élément d'un sous-groupe abélien non dénombrable de $\operatorname{Bir}\left(\mathbb{P}^{2}(\mathbb{C})\right)$ laisse invariant un champ de vecteurs rationnel non nul. Ceci permet d'utiliser les travaux de Cantat et Favre sur les symétries des feuilletages des surfaces [CF03] ; nous en déduisons le :

ThÉorème 1.5. Si G est un sous-groupe abélien maximal non dénombrable de $\operatorname{Bir}\left(\mathbb{P}^{2}(\mathbb{C})\right.$ ), il vérifie l'une des propriétés suivantes:

- G possède des éléments de torsion ;

- G est conjugué à un sous-groupe de J. 


\section{Automorphismes Du Groupe De Cremona}

Dans le but de montrer en particulier qu'à conjugaison près $\varphi$ laisse le groupe de Jonquières invariant, nous sommes amenés à étudier les sous-groupes abéliens maximaux non dénombrables de J :

ThÉORÈme 1.6. Soit G un sous-groupe abélien maximal non dénombrable du groupe de Jonquières. Le groupe G satisfait l'une des propriétés suivantes :

- G contient un élément de torsion ;

- tout sous-groupe de $\operatorname{Bir}\left(\mathbb{P}^{2}(\mathbb{C})\right)$ agissant par conjugaison sur $\mathrm{G}$ est virtuellement résoluble ;

- $\mathrm{G}$ est conjugué à $\mathrm{J}_{a}=\{(x+P(y), y) \mid P \in \mathbb{C}(y)\}$;

- $\mathrm{G}$ est conjugué à $\mathrm{T}=\{(x+\alpha, y+\beta) \mid \alpha, \beta \in \mathbb{C}(y)\}$.

Remarque 1.7. Voici deux propriétés qui permettent de distinguer $\mathrm{J}_{a}$ des autres sous-groupes abéliens maximaux de $\mathrm{J}$ :

- le groupe $\mathrm{J}_{a}$ ne contient pas d'élément de torsion ;

- le groupe non résoluble $\left\{(a(y) x+b(y), \nu(y)) \mid a \in \mathbb{C}(y)^{*}, b \in \mathbb{C}(y), \nu \in \operatorname{PGL}(2, \mathbb{C})\right\}$ agit par conjugaison sur $\mathrm{J}_{a}$.

Les théorèmes 1.5 et 1.6 ajoutés à la remarque 1.7 assurent que si $\varphi$ désigne un automorphisme du groupe de Cremona, alors $\varphi\left(\mathrm{J}_{a}\right)$ est conjugué à $\mathrm{J}_{a}$; par suite, quitte à composer $\varphi$ par un automorphisme intérieur de $\operatorname{Bir}\left(\mathbb{P}^{2}(\mathbb{C})\right)$, nous avons $\varphi\left(\mathrm{J}_{a}\right)=\mathrm{J}_{a}$. Ensuite nous montrons qu'à conjugaisons près, les groupes

$$
\begin{array}{cl}
\mathrm{T}_{1}=\{(x+\alpha, y) \mid \alpha \in \mathbb{C}\}, & \mathrm{T}_{2}=\{(x, y+\alpha) \mid \alpha \in \mathbb{C}\}, \\
\mathrm{D}_{1}=\left\{(\alpha x, y) \mid \alpha \in \mathbb{C}^{*}\right\}, & \mathrm{D}_{2}=\left\{(x, \alpha y) \mid \alpha \in \mathbb{C}^{*}\right\}
\end{array}
$$

sont invariants $\operatorname{par} \varphi$ et ce sans modifier le fait que $J_{a}$ soit fixé $\operatorname{par} \varphi$. Ainsi $\varphi$ induit deux automorphismes du groupe des transformations affines de la droite : celui engendré par $T_{1}$ et $D_{1}$ et celui engendré par $T_{2}$ et $\mathrm{D}_{2}$. Or nous avons le résultat suivant, sans doute bien connu, dont on peut trouver une preuve dans [Dés06] :

LEMme 1.8. Si $\phi$ est un automorphisme du groupe des transformations affines de la droite complexe, $\phi$ est la composée d'un automorphisme intérieur et de l'action d'un isomorphisme du corps $\mathbb{C}$.

Il s'en suit que, quitte à composer $\varphi$ par un automorphisme intérieur et un isomorphisme du corps $\mathbb{C}$, les groupes $\mathrm{T}=\{(x+\alpha, y+\beta) \mid \alpha, \beta \in \mathbb{C}\}$ et $\mathrm{D}=\left\{(\alpha x, \beta y) \mid \alpha, \beta \in \mathbb{C}^{*}\right\}$ sont laissés invariants point par point. Nous constatons après ces modifications que les involutions $(x, 1 / y)$, $(y, x)$ et $(1 / x, 1 / y)$ sont fixées par $\varphi$. Or le groupe engendré par $\mathrm{D}, \mathrm{T},(y, x)$ et $(x, 1 / y)$ contient $\operatorname{PGL}(3, \mathbb{C}) ;$ ces groupes et involutions étant invariants point par point par $\varphi$, nous obtenons grâce au théorème 1.2 le résultat annoncé.

\section{Feuilletages}

\subsection{Lemme fondamental}

Introduisons quelques notations et définitions. Si $S$ est une surface complexe compacte, un feuilletage $\mathcal{F}$ sur $S$ est donné par une famille $\left(X_{i}\right)_{i}$ de champs de vecteurs holomorphes à zéros isolés définis sur les ouverts $\mathcal{U}_{i}$ d'un recouvrement de $S$. Les champs $X_{i}$ sont soumis à des conditions de compatibilité : il existe $g_{i j}$ dans $\mathcal{O}^{*}\left(\mathcal{U}_{i} \cap \mathcal{U}_{j}\right)$ tel que $X_{i}$ coïncide avec $g_{i j} X_{j}$ sur $\mathcal{U}_{i} \cap \mathcal{U}_{j}$. Notons qu'un champ de vecteurs méromorphe non trivial sur $S$ définit un tel feuilletage. Soit $S$ une surface projective munie d'un feuilletage $\mathcal{F}$; nous désignons $\operatorname{par} \operatorname{Bir}(S, \mathcal{F})$ (respectivement $\operatorname{Aut}(S, \mathcal{F})$ ) le groupe des 


\section{J. DÉSERTI}

transformations birationnelles (respectivement holomorphes) laissant le feuilletage $\mathcal{F}$ invariant sur la surface $S$.

Le lemme qui suit s'avère fondamental ; il repose sur un argument classique de géométrie analytique.

Lemme 2.1. Soit G un sous-groupe abélien non dénombrable du groupe de Cremona. Pour tout élément $f$ de $G$ il existe au moins un champ de vecteurs méromorphe non nul $X$ sur $\mathbb{P}^{2}(\mathbb{C})$ tel que $f_{*} X=X$. En particulier, tout élément $f$ de G préserve au moins un feuilletage holomorphe singulier de $\mathbb{P}^{2}(\mathbb{C})$.

Démonstration. Rappelons que le degré d'une transformation birationnelle $f=\left[P_{0}: P_{1}: P_{2}\right]$ est égal au degré des $P_{i}$. Puisque le groupe $\mathrm{G}$ n'est pas dénombrable, il existe un entier $n$ tel que $\mathrm{G}_{n}=\{f \in \mathrm{G} \mid \operatorname{deg} f=n\}$ ne soit pas dénombrable. Par suite, l'adhérence de Zariski $\overline{\mathrm{G}_{n}}$ de $\mathrm{G}_{n}$ dans

$$
E_{n}=\left\{f \in \operatorname{Bir}\left(\mathbb{P}^{2}(\mathbb{C})\right) \mid \operatorname{deg} f \leqslant n\right\}
$$

est un ensemble algébrique de dimension supérieure ou égale à un. Considérons un élément $f_{0}$ dans $\mathrm{G}_{n}$ tel que la dimension du germe $\left(\overline{\mathrm{G}_{n}}, f_{0}\right)$ soit supérieure ou égale à un et $f_{0}$ n'appartienne pas au lieu singulier de $\overline{\mathrm{G}_{n}}$. D'après le théorème des fonctions implicites, il existe une application analytique non constante $s \mapsto f_{s}$ du disque unité $\mathbb{D}$ dans $\overline{\mathrm{G}_{n}}$ telle que $f_{s=0}=f_{0}$ et $\partial f_{s} /\left.\partial s\right|_{s=0} \neq 0$. Si $m$ n'appartient pas au lieu d'indétermination de $f_{0}^{-1}$ posons :

$$
X(m)=\left.\frac{\partial f_{s}}{\partial s}\right|_{s=0}\left(f_{0}^{-1}(m)\right) .
$$

Ce champ $X$ s'étend en un champ de vecteurs rationnel sur le plan projectif. Puisque $\partial f_{s} /\left.\partial s\right|_{s=0}$ est non nul, le champ $X$ n'est pas identiquement nul. Remarquons que les éléments de $\overline{\mathrm{G}_{n}}$ sont des applications rationnelles qui commutent; en particulier $f_{s}$ et $f_{0}$ commutent pour tout $s$ dans $\mathbb{D}$. En dérivant l'identité $f_{0} f_{s} f_{0}^{-1}(m)=f_{s}(m)$ par rapport à $s$ à $m$ fixé, nous obtenons $f_{0 *} X=X$.

$A$ posteriori, si $g$ est un élément quelconque de $\mathrm{G}$, nous constatons, en considérant le chemin $g_{s}=g f_{0}^{-1} f_{s}$, que $g$ laisse un champ rationnel invariant.

Le lemme fondamental nous permet d'établir la :

Proposition 2.2. Si G est un sous-groupe abélien non dénombrable de $\operatorname{Bir}\left(\mathbb{P}^{2}(\mathbb{C})\right)$, G vérifie l'une des conditions suivantes :

- tous les éléments de $\mathrm{G}$ sont périodiques ;

- G laisse un feuilletage invariant.

Démonstration. Soit $f$ un élément non trivial de $\mathrm{G}$; notons $\chi(f)$ le $\mathbb{C}$-espace vectoriel défini par :

$$
\chi(f)=\left\{X \text { champ de vecteurs rationnel } \mid f_{*} X=X\right\} .
$$

Si la dimension de $\chi(f)$ est 1 , alors $\chi(f)=\mathbb{C}$. $X$ et, pour tout $g$ dans $\mathrm{G}$, nous avons $g_{*} X=\lambda(g) X$ où $\lambda(g)$ désigne un élément de $\mathbb{C}^{*}$ : le feuilletage induit par $X$ est donc invariant par $\mathrm{G}$.

Supposons que $\chi(f)$ soit de dimension deux. Si pour tout $Y$ et $\tilde{Y}$ dans $\chi(f)$ il existe une fonction rationnelle $R$ telle que $Y=R \tilde{Y}$, notons $X$ un élément fixé de $\chi(f)$; pour tout $g$ dans $\mathrm{G}$, le champ $g_{*} X$ s'écrit $\mu(g) X$, où cette fois $\mu(g)$ est rationnelle. Le feuilletage induit par $X$ est donc invariant par $\mathrm{G}$. Sinon nous obtenons un morphisme $\nu: \mathrm{G} \rightarrow \mathrm{GL}(\chi(f)) \simeq \mathrm{GL}(2, \mathbb{C})$ tel que $g_{*} X=\nu(g) X$. L'image de $\mathrm{G}$ est abélienne donc triangulable ; il s'en suit l'existence d'un vecteur propre commun à tous les $\nu(g)$ produisant un feuilletage invariant par $\mathrm{G}$.

Supposons désormais que la dimension de $\chi(f)$ soit supérieure ou égale à trois ; choisissons $X_{1}$, $X_{2}$ et $X_{3}$ trois éléments linéairement indépendants dans $\chi(f)$. Il existe $\varphi_{1}$ et $\varphi_{2}$ deux fonctions 


\section{Automorphismes du Groupe De Cremona}

rationnelles non toutes constantes telles que $\varphi_{1} X_{1}+\varphi_{2} X_{2}+X_{3}=0$. Supposons par exemple que $\varphi_{1}$ soit non constante ; à partir de $X_{3}=-\varphi_{1} X_{1}-\varphi_{2} X_{2}$ nous obtenons que $\varphi_{1}$ est invariant par $f$. Nous avons alors l'alternative suivante : ou bien $f$ laisse plusieurs fonctions invariantes auquel cas $f$ est périodique, ou bien $f$ laisse une unique fonction invariante alors, par abélianité, G laisse une fibration invariante.

Dorénavant nous supposerons que G est un sous-groupe abélien maximal non dénombrable du groupe de Cremona et que $\mathcal{F}$ est un feuilletage de $\mathbb{P}^{2}(\mathbb{C})$ invariant sous l'action de $\mathrm{G}$.

\subsection{Symétrie des feuilletages}

Dans cette partie nous allons montrer le :

ThÉorÈme 2.3. Soit $\mathrm{G}$ un sous-groupe abélien maximal non dénombrable de $\operatorname{Bir}\left(\mathbb{P}^{2}(\mathbb{C})\right)$. Le groupe $\mathrm{G}$ satisfait l'une des conditions suivantes :

- G possède un élément de torsion ;

- G est conjugué à un sous-groupe du groupe de Jonquières.

La preuve repose en particulier sur certains résultats de Cantat et Favre (voir [CF03]).

2.2.1 Automorphismes des surfaces minimales. Supposons qu'il existe une surface $S$ et une transformation birationnelle $\xi$ de $S$ dans $\mathbb{P}^{2}(\mathbb{C})$ telles que $\operatorname{Aut}\left(S, \xi^{*} \mathcal{F}\right)=\operatorname{Bir}\left(S, \xi^{*} \mathcal{F}\right)$. Puisque $\operatorname{Aut}\left(S, \xi^{*} \mathcal{F}\right)$ est infini, alors, d'après Cantat et Favre [CF03, théorème 1.1], nous pouvons nous ramener à l'une des situations suivantes :

- $\xi^{*} \mathcal{F}$ est invariant par un champ de vecteurs holomorphe sur $S$;

- $\xi^{*} \mathcal{F}$ est une fibration elliptique.

Dans notre contexte, le groupe G n'étant pas dénombrable le dernier cas n'arrive pas [BHPV04, Can99]. Nous pouvons donc supposer que $\xi^{*} \mathcal{F}$ est invariant par un champ holomorphe $X$ sur $S$. Il existe alors, toujours d'après [CF03], un morphisme birationnel $\psi$ de $S$ vers un modèle minimal $\tilde{S}$ de $S$ tel que $\tilde{\mathrm{G}}=\psi g \psi^{-1}(\mathrm{G})$ soit un sous-groupe de $\operatorname{Aut}(\tilde{S})$.

Les surfaces rationnelles minimales sont $\mathbb{P}^{1}(\mathbb{C}) \times \mathbb{P}^{1}(\mathbb{C}), \mathbb{P}^{2}(\mathbb{C})$ et les surfaces de Hirzebruch $\mathrm{F}_{n}$, $n \geqslant 2$. Pour $n \geqslant 2$, le groupe des automorphismes de la $n$-ième surface de Hirzebruch $\mathrm{F}_{n}$ est, dans la carte affine $(x, y)$, du type :

$$
\left\{\left(\frac{\alpha x+f(y)}{(c y+d)^{n}}, \frac{a y+b}{c y+d}\right) \mid\left(\begin{array}{ll}
a & b \\
c & d
\end{array}\right) \in \operatorname{PGL}(2, \mathbb{C}), \alpha \in \mathbb{C}^{*}, f \in \mathbb{C}[y], \operatorname{deg} f \leqslant n\right\} .
$$

Notons $\mathrm{J}_{a, n}=\{(x+P(y), y) \mid P \in \mathbb{C}[y], \operatorname{deg} P \leqslant n\}, \mathrm{T}=\{(x+\alpha, y+\beta) \mid \alpha, \beta \in \mathbb{C}\}$ le groupe des translations, $\mathrm{D}=\left\{(\alpha x, \beta y) \mid \alpha, \beta \in \mathbb{C}^{*}\right\}$ le groupe diagonal, $\mathrm{D}_{1}$ le groupe $\left\{(\alpha x, y) \mid \alpha \in \mathbb{C}^{*}\right\}$ et $\pi: \operatorname{Aut}\left(\mathrm{F}_{n}\right) \rightarrow \operatorname{PGL}(2, \mathbb{C})$ la seconde projection. Si $H$ désigne un sous-groupe de Aut $\left(\mathrm{F}_{n}\right)$, posons $H_{0}=H \cap \operatorname{ker} \pi$. Rappelons que si $H$ est un sous-groupe abélien de $\operatorname{PGL}(2, \mathbb{C})$, alors $H$ est, à conjugaison près, un groupe de translations, d'homothéties ou le groupe à quatre éléments $\{y,-y, 1 / y,-1 / y\}$.

Remarque 2.4. Le groupe $\mathrm{J}_{a, n}$ est un sous-groupe abélien maximal de $\operatorname{Aut}\left(\mathrm{F}_{n}\right)$. En effet, un élément $g$ de $\operatorname{Aut}\left(\mathrm{F}_{n}\right)$ commute à $(x+1, y)$ si et seulement s'il s'écrit $(x+f(y), a y+b)$; la commutation de $g$ et $(x+y, y)$ implique alors que $g$ appartient à $\mathrm{J}_{a, n}$.

Proposition 2.5. Soient $S$ une surface rationnelle minimale et $H$ un sous-groupe abélien maximal non dénombrable de $\operatorname{Aut}(S)$. Alors nous sommes dans l'une des situations suivantes :

- $H$ contient un élément de torsion ;

- $H$ coïncide, à conjugaison dans $\operatorname{Aut}(S)$ près, avec $\mathrm{J}_{a, n}$; 


\section{J. DÉSERTI}

- $H$ est, à conjugaison dans $\operatorname{Aut}(S)$ près, le groupe des translations ;

- $H$ est contenu, à conjugaison birationnelle près, dans $\left\{(\alpha x+\beta, \gamma y) \mid \alpha, \gamma \in \mathbb{C}^{*}, \beta \in \mathbb{C}\right\}$.

Avant de donner la preuve de la proposition 2.5 mentionnons le :

Corollaire 2.6. Soient $S$ une surface rationnelle minimale et $H$ un sous-groupe abélien non dénombrable de $\operatorname{Aut}(S)$ maximal dans $\operatorname{Bir}(S)$. Alors $H$ vérifie, à conjugaison près, l'une des conditions suivantes :

- $H$ contient un élément de torsion ;

- $H$ coïncide avec $\mathrm{J}_{a, n}$;

- $H$ est le groupe des translations.

La preuve de la proposition 2.5 est longue ; nous raisonnons suivant la nature de $S$ puis celle du sous-groupe abélien $\pi(H)$ de $\operatorname{PGL}(2, \mathbb{C})$.

Démonstration. Le cas $S=\mathbb{P}^{1}(\mathbb{C}) \times \mathbb{P}^{1}(\mathbb{C})$ est élémentaire car son groupe d'automorphismes est $(\operatorname{PGL}(2, \mathbb{C}) \times \operatorname{PGL}(2, \mathbb{C})) \rtimes(y, x)$.

Si $S=\mathbb{P}^{2}(\mathbb{C})$, alors $\operatorname{Aut}(S)=\operatorname{PGL}(3, \mathbb{C})$ donc $H$ est, à conjugaison près, l'un des groupes suivants :

$$
\mathrm{D}, \quad \mathrm{T}, \quad\left\{(x+\alpha, \beta y) \mid \alpha \in \mathbb{C}, \beta \in \mathbb{C}^{*}\right\}, \quad\left\{(\alpha x+\beta y, \alpha y) \mid \alpha \in \mathbb{C}^{*}, \beta \in \mathbb{C}\right\} ;
$$

remarquons que si $H$ n'est pas conjugué à $\mathrm{T}$, il contient un élément de torsion.

Désormais $S$ est une surface de Hirzebruch $\mathrm{F}_{n}$ avec $n \geqslant 2$; nous allons raisonner en distinguant les cas où $\pi(H)$ est trivial, fini non trivial ou infini.

Lemme 2.7. Soit $H$ un sous-groupe abélien maximal non dénombrable de Aut $\left(\mathrm{F}_{n}\right)$ tel que $\pi(H)$ soit trivial. Alors $H$ coïncide avec $\mathrm{J}_{a, n}$.

Démonstration. Deux applications $(\alpha x+P(y), y)$ et $(\beta x+Q(y), y)$ commutent si et seulement si $(\alpha-1) Q(y)=(\beta-1) P(y)$. Si $H$ contient un élément non trivial du type $(x+P(y), y)$, alors $H$ est contenu dans $\mathrm{J}_{a, n}$; par maximalité (remarque 2.4), $H$ et $\mathrm{J}_{a, n}$ cö̈ncident. Sinon soit $f=(\alpha x+P(y), y)$ avec $\alpha$ différent de 1 ; à conjugaison près par $(x+P(y) /(1-\alpha), y)$, la transformation $f$ s'écrit $(\alpha x, y)$. Après cette modification, nous avons par abélianité $H=\mathrm{D}_{1}$ mais le groupe $\mathrm{D}_{1}$ n'est pas maximal.

Lemme 2.8. Soit $H$ un sous-groupe abélien maximal non dénombrable de Aut $\left(\mathrm{F}_{n}\right)$ tel que $\pi(H)$ soit fini non trivial. Alors $H$ contient au moins un élément de torsion.

Démonstration. L'étude du cas où $\pi(H)$ est trivial assure que $H_{0}$ est, à conjugaison près, un sousgroupe de $\mathrm{J}_{a, n}$ ou $\mathrm{D}_{1}$.

Considérons le cas où $\pi(H)$ est, à conjugaison près, le groupe $\{y,-y, 1 / y,-1 / y\}$. Remarquons qu'alors les éléments de $H \backslash H_{0}$ sont de la forme $(\alpha x+f(y),-y)$ ou $\left((\alpha x+f(y)) / y^{n}, 1 / y\right)$ (voir (1)). Puisque $H$ n'est pas dénombrable, il en est de même pour $H_{0}$.

Supposons que $H_{0}$ soit contenu dans $\mathrm{D}_{1}$. Un élément $(\alpha x, y)$ de $H_{0}$ commute à $\left((\gamma x+R(y)) / y^{n}, 1 / y\right)$ si et seulement si $(\alpha-1) R(y)$ est nul ; puisque $H_{0}$ est non dénombrable, $R$ est nul. De même, par abélianité de $H$, nous obtenons que tout élément $g$ de $H$ tel que $\pi(g)=-y$ s'écrit $(\beta x,-y)$. Ainsi, par maximalité, $H_{0}$ et $\mathrm{D}_{1}$ coïncident ; le groupe $H$ contient donc des éléments de torsion.

Supposons que $H_{0}$ soit un sous-groupe de $\mathrm{J}_{a, n}$. Soit $f=(x+Q(y), y)$ dans $H_{0} \backslash\{\mathrm{id}\}$. Le carré d'un élément $g$ de $H$ tel que $\pi(g)=-y$ appartient à $H_{0}$; en particulier $g$ s'écrit $(\beta x+P(y),-y)$ 


\section{Automorphismes Du Groupe De Cremona}

avec $\beta= \pm 1$. Si $\beta$ vaut 1 (respectivement -1 ), la commutation de $g$ avec $f$ implique que $Q$ est pair (respectivement impair). Le groupe $H_{0}$ étant non trivial ou bien tous les éléments $h$ de $H$ tels que $\pi(h)=-y$ sont du type $(x+P(y),-y)$, ou bien du type $(-x+P(y),-y)$. Supposons par exemple que nous soyons dans la première éventualité. Ecrivons $g$ sous la forme $\left(x+P_{1}\left(y^{2}\right)+y P_{2}\left(y^{2}\right),-y\right)$; alors $g^{2}=\left(x+2 P_{1}\left(y^{2}\right), y\right)$ appartient à $H_{0}$. En utilisant la maximalité de $H$ on peut vérifier que $\left(x-P_{1}\left(y^{2}\right), y\right)$ est aussi dans $H_{0}$ (il suffit de constater que tout élément dont la projection par $\pi$ est $1 / y$ et qui commute à $g^{2}$ commute à $\left(x-P_{1}\left(y^{2}\right), y\right)$ ). Par suite l'élément périodique $\left(x+y P_{2}\left(y^{2}\right),-y\right)$ est dans $H$. Un raisonnement analogue permet de conclure lorsque tous les éléments $h$ de $H$ satisfaisant $\pi(h)=-y$ sont de la forme $(-x+P(y),-y)$. Le cas $\beta=-1$ se traite de la même façon.

Etudions le cas où $\pi(H)$ est engendré par un élément $\alpha y$ d'ordre fini $q$. Si $H_{0}$ est un sous-groupe non dénombrable de $\mathrm{D}_{1}$, alors $H$ n'est pas maximal : comme précédemment nous obtenons que $H$ est un sous-groupe strict de D.

Supposons que $H_{0}$ soit un sous-groupe non dénombrable de $\mathrm{J}_{a, n}$. Les éléments $g=(\gamma x+R(y), \alpha y)$ et $f=\left(x+p_{0}+p_{1} y+\cdots+p_{n} y^{n}, y\right)$ commutent si et seulement si $\left(\gamma-\alpha^{j}\right) p_{j}$ est nul pour tout $j$ compris entre 0 et $n$; puisque $H_{0}$ est non trivial, il existe un entier $\ell$ tel que $\gamma=\alpha^{\ell}$. La condition de commutation assure que $p_{j}$ ne peut être non nul que s'il existe un entier $m$ tel que $j=\ell+m q$; par maximalité $H_{0}=\left\{\left(x+y^{\ell} P\left(y^{q}\right), y\right) \mid P \in \mathbb{C}[y],(\operatorname{deg} P) q+\ell \leqslant n\right\}$. Quitte à composer $g$ par un élément de $H_{0}$, il s'écrit :

$$
\left(\alpha^{\ell} x+r_{0}\left(y^{q}\right)+y r_{1}\left(y^{q}\right)+\cdots+y^{\ell-1} r_{\ell-1}\left(y^{q}\right)+y^{\ell+1} r_{\ell+1}\left(y^{q}\right)+\cdots+y^{q-1} r_{q-1}\left(y^{q}\right), \alpha y\right) ;
$$

une telle transformation est périodique de période $q$.

Lemme 2.9. Soit $H$ un sous-groupe abélien maximal non dénombrable de Aut $\left(\mathrm{F}_{n}\right), n \geqslant 2$, tel que $\pi(H)$ soit infini. Alors $H$ vérifie l'une des propriétés suivantes :

- $H$ contient un élément de torsion ;

- $H$ est contenu dans $\left\{(\alpha x+\beta, y+\gamma) \mid \alpha \in \mathbb{C}^{*}, \beta, \gamma \in \mathbb{C}\right\}$ à conjugaison birationnelle près.

Démonstration. L'étude du cas $\pi(H)$ trivial assure que $H_{0}$ est, à conjugaison près, un sous-groupe de $\mathrm{J}_{a, n}$ ou $\mathrm{D}_{1}$.

Supposons que $\pi(H)$ soit, à conjugaison près, un groupe infini de racines de l'unité. Notons qu'alors, toujours d'après (1), les éléments de $H \backslash H_{0}$ s'écrivent $(\alpha x+f(y), \beta y)$. Ici encore $H_{0}$ n'est pas dénombrable.

Si $H_{0}$ est un sous-groupe de $\mathrm{D}_{1}$, nous pouvons choisir un élément d'ordre infini $(\lambda x, y)$ dans $H_{0}$; il commute à la transformation $(t x+P(y), \beta y)$ de $H \backslash H_{0}$ si et seulement si $P$ est nul. Ainsi $H$ est un sous-groupe du groupe diagonal et n'est donc pas maximal (dénombrabilité de $\pi(H)$ ).

Supposons que $H_{0}$ soit un sous-groupe de $\mathrm{J}_{a, n}$; soient $f=\left(x+p_{0}+p_{1} y+\cdots+p_{n} y^{n}, y\right)$ dans $H_{0} \backslash$ id $\}$ et $g=(\alpha x+a(y), \beta y)$ dans $H \backslash H_{0}$. Les éléments $f$ et $g$ commutent si et seulement si $\left(\alpha-\beta^{j}\right) p_{j}$ est nul pour tout $j$ compris entre 0 et $n$. Comme $\pi(H)$ est infini, nous pouvons choisir $g$ de sorte que $\beta$ soit d'ordre strictement supérieur à $n$; par suite il existe un unique entier $k$ compris entre 0 et $n$ tel que $\alpha-\beta^{k}$ soit nul. Nous en déduisons que $H_{0}$ est un sous-groupe de $\left\{\left(x+\varepsilon y^{k}, y\right) \mid \varepsilon \in \mathbb{C}\right\}$ et que $g$ est du type $\left(\beta^{k} x+a(y), \beta y\right)$; par maximalité, $H_{0}$ et $\left\{\left(x+\varepsilon y^{k}, y\right) \mid \varepsilon \in \mathbb{C}\right\}$ coïncident. Quitte à composer $g$ par un élément de $H_{0}$, le coefficient d'ordre $k$ de $a$ est nul. Notons $q$ la période de $\beta$; alors $g^{q}$ est de la forme $\left(x+\varepsilon y^{k}, y\right)$. Mais $\varepsilon$ est nécessairement nul puisque le coefficient d'ordre $k$ de $a$ est nul ; autrement dit $H$ possède un élément de torsion.

Supposons que $\pi(H)$ soit un groupe de translations ; nous pouvons nous ramener au cas où $y+1$ appartient à $\pi(H)$. Considérons dans $H$ un élément $f$ du type $(t x+P(y), y+1)$; écrivons $P$ sous la forme $: p_{0}+p_{1} y+\cdots+p_{n} y^{n}$. 


\section{J. DÉSERTI}

Si $t$ est différent de 1, posons $q_{n}=p_{n} /(t-1)$ et pour $j \leqslant n-1$ :

$$
q_{j}=\frac{1}{t-1}\left(p_{j}+\sum_{k=j+1}^{n} \complement_{k}^{j} q_{k}\right) .
$$

Quitte à conjuguer $f \operatorname{par}\left(x+\sum_{j=0}^{n} q_{j} y^{j}, y\right)$, nous avons $f=(t x, y+1)$. Un élément $(s x+R(y), y+\gamma)$ de $H$ commute à $f$ si et seulement si $R$ est nul ; par suite, $H$ et $\left\{(\alpha x, y+\beta) \mid \alpha \in \mathbb{C}^{*}, \beta \in \mathbb{C}\right\}$ coïncident donc $H$ contient des éléments périodiques.

Si $t$ vaut un, posons $q_{n}=-p_{n} /(n+1)$ et pour tout $j$ compris entre 0 et $n-1$ :

$$
q_{j}=-\frac{1}{j+1}\left(p_{j}+\sum_{k=j+1}^{n} \complement_{k+1}^{j} q_{k}\right) \text {. }
$$

A conjugaison birationnelle près par $\left(x+\sum_{j=0}^{n} q_{j} y^{j+1}, y\right)$, la transformation $f$ s'écrit $(x, y+1)$. Un élément de $H$ qui commute à $f$ est du type $(s x+\mu, y+\lambda)$. Par suite, $H$ est contenu, à conjugaison birationelle près, dans $\left\{(\alpha x+\beta, y+\gamma) \mid \alpha \in \mathbb{C}^{*}, \beta, \gamma \in \mathbb{C}\right\}$.

Finalement étudions le cas où $\pi(H)$ est un sous-groupe d'homothéties contenant un élément $\lambda y$ d'ordre infini. Soit $f=\left(t x+p_{0}+p_{1} y+\cdots+p_{n} y^{n}, \lambda y\right)$ dans $H$; si, pour tout $j$ compris entre 0 et $n$, le terme $t-\lambda^{j}$ est non nul, alors, quitte à conjuguer $f$ par

$$
\left(x+\sum_{j=0}^{n} \frac{p_{j}}{t-\lambda^{j}} y^{j}, y\right),
$$

$f$ est de la forme $(t x, \lambda y)$. Un élément de $H$ qui commute à $(t x, \lambda y)$ est du type $(s x+\eta, \mu y)$; autrement dit $H$ est un sous-groupe abélien maximal non dénombrable de :

$$
\left\{(\alpha x+\beta, \gamma y) \mid \alpha, \gamma \in \mathbb{C}^{*}, \beta \in \mathbb{C}\right\} .
$$

Par suite, $H$ coïncide, à conjugaison près, avec $\left\{(x+\alpha, \beta y) \mid \alpha \in \mathbb{C}, \beta \in \mathbb{C}^{*}\right\}$ ou avec le groupe diagonal ; ces deux groupes contiennent des éléments de torsion.

Reste à traiter le cas où il existe un entier $j_{0}$ tel que $t=\lambda^{j_{0}}$; puisque $\lambda$ n'est pas racine de l'unité, $j_{0}$ est unique. Quitte à conjuguer $f$ par :

$$
\left(x+\sum_{\substack{j=0 \\ j \neq j_{0}}}^{n} \frac{p_{j}}{t-\lambda^{j}} y^{j}, y\right)
$$

et par l'homothétie $\left(x, p_{j_{0}} y\right)$ si $p_{j_{0}}$ est non nul, nous pouvons supposer que $f$ s'écrit $\left(\lambda^{j_{0}} x+v y^{j_{0}}, \lambda y\right)$ où $v \in\{0,1\}$. Un calcul montre qu'un élément $g$ de $H$ commute à $f$ si et seulement si $g=$ $\left(\beta^{j_{0}} x+v^{\prime} y^{j_{0}}, \beta y\right)$. Donc $H=\left\{\left(\alpha^{j_{0}} x+\varepsilon y^{j_{0}}, \alpha y\right) \mid \alpha \in \mathbb{C}^{*}, \varepsilon \in \mathbb{C}\right\}$; notons que $H$ contient des éléments de torsion.

Ces trois lemmes épuisent tous les cas possibles lorsque $S$ est une surface de Hirzebruch ; ceci achève la démonstration de la proposition.

2.2.2 Cas restants. Lorsque la situation $\S$ 2.2.1 n'a pas lieu, Cantat et Favre [CF03, théorème 1.2 et exemple 1.3], assurent que nous avons, à conjugaison près, l'alternative suivante :

- il existe des entiers $p, q, r$ et $s$ tels que, à revêtement fini près, nous ayons :

$$
\operatorname{Bir}\left(\mathbb{P}^{2}(\mathbb{C}), \mathcal{F}\right)=\left\{\left(x^{p} y^{q}, x^{r} y^{s}\right),(\alpha x, \beta y) \mid \alpha, \beta \in \mathbb{C}^{*}\right\},
$$

- $\mathcal{F}$ est une fibration rationnelle. 


\section{Automorphismes Du Groupe De Cremona}

Dans la première éventualité, un sous-groupe abélien maximal non dénombrable $\mathrm{G}$ de $\operatorname{Bir}\left(\mathbb{P}^{2}(\mathbb{C}), \mathcal{F}\right)$ est le groupe diagonal ou le groupe suivant :

$$
\left\{\left(x^{p} y^{q}, x^{r} y^{s}\right),(\alpha x, \beta y) \mid \alpha, \beta \in \mathbb{C}^{*}, \alpha=\alpha^{p} \beta^{q}, \beta=\alpha^{r} \beta^{s}\right\} .
$$

Si G est conjugué à D, il contient des éléments de torsion. Etudions le second cas ; puisque G n'est pas dénombrable, il ne peut se réduire au groupe engendré par $\left(x^{p} y^{q}, x^{r} y^{s}\right)$. Il existe donc un élément non trivial dans $\mathrm{G}$ du type $(\lambda x, \mu y)$ satisfaisant $\lambda=\lambda^{p} \mu^{q}$ et $\mu=\lambda^{r} \mu^{s}$; pour tout $\rho$, la transformation $\left(\lambda^{\rho} x, \mu^{\rho} y\right)$ vérifie ces égalités donc appartient à G. Soit $\rho$ tel que $\lambda^{\rho}=\mathrm{i}$; alors $\mu^{\rho}=\exp (\mathrm{i} \pi((1-p) / 2 q))$ est aussi racine de l'unité : $\left(\lambda^{\rho} x, \mu^{\rho} y\right)$ est un élément de torsion de $\mathrm{G}$. Le groupe $\mathrm{G}$ contient donc des éléments de torsion de tout ordre.

Reste à considérer le cas où $\mathrm{G}$ laisse une fibration rationnelle invariante, i.e. est, à conjugaison près, un sous-groupe du groupe de Jonquières : c'est l'objet du $\S 4$.

\section{Le groupe $\mathbf{J}_{0}$}

\subsection{Préliminaires}

Notons $\pi$ la projection de $\mathrm{J} \simeq \operatorname{PGL}(2, \mathbb{C}(y)) \rtimes \operatorname{PGL}(2, \mathbb{C})$ sur $\operatorname{PGL}(2, \mathbb{C})$ et $\mathrm{G}_{0}$ le groupe $(\operatorname{ker} \pi) \cap \mathrm{G}$; remarquons que $\mathrm{J}_{0}=\operatorname{ker} \pi$ est isomorphe à $\operatorname{PGL}(2, \mathbb{C}(y))$. Si $f_{S}$ est une famille d'éléments de $\mathrm{G}$, alors $\left\langle f_{S}\right\rangle$ est le groupe engendré par la famille $f_{S}$.

Introduisons les quatre types de sous-groupes abéliens de $\mathrm{J}_{0}$ suivants :

- $\mathrm{J}_{m}=\left\{(a(y) x, y) \mid a \in \mathbb{C}(y)^{*}\right\}$,

- pour tout $F$ dans $\mathbb{C}(y)$ qui n'est pas un carré :

$$
\mathrm{J}_{F}=\left\{\left(\frac{a(y) x+F(y)}{x+a(y)}, y\right) \mid a \in \mathbb{C}(y)\right\},
$$

- pour tous $C$ et $F$ dans $\mathbb{C}(y)$ tels que $F$ ne soit pas un carré :

$$
\mathrm{I}_{F}^{C}=\left\langle\left(\frac{F(y)}{x}, y\right),\left(\frac{C(y) x-F(y)}{x-C(y)}, y\right)\right\rangle
$$

- et enfin pour tout $B$ dans $\mathbb{C}(y)^{*}$ :

$$
\mathrm{I}_{B}=\left\langle(-x, y),\left(\frac{1}{B(y) x}, y\right)\right\rangle .
$$

Remarques 3.1.

- Si $F$ était un carré, le groupe $\mathrm{J}_{F}$ correspondant serait conjugué à $\mathrm{J}_{m}$.

- Les groupes $\mathrm{I}_{F}^{C}$ et $\mathrm{I}_{B}$ ont quatre éléments.

- Chaque élément du groupe $\mathrm{J}_{F}$ laisse la courbe hyperelliptique $x^{2}=F(y)$ invariante ; une façon de distinguer ces différents groupes est de considérer leurs courbes de points fixes. Par analogie avec $\operatorname{PGL}(2, \mathbb{R})$, le groupe $\mathrm{J}_{a}$ est dit parabolique, $\mathrm{J}_{m}$ hyperbolique et $\mathrm{J}_{F}$ elliptique.

Dans la suite, $\psi(a, b, c, d ; \nu(y))$ désigne l'élément $((a(y) x+b(y)) /(c(y) x+d(y)), \nu(y))$ du groupe de Jonquières.

\subsection{Centralisateurs}

Comme souvent en théorie des groupes, des calculs effectifs de centralisateurs s'avèrent nécessaires.

Lemme 3.2. Le centralisateur dans $\mathrm{J}_{0}$ d'un élément non trivial de $\mathrm{J}_{a}$ est $\mathrm{J}_{a}$.

Le centralisateur dans $\mathrm{J}_{0}$ d'un élément non trivial de $\mathrm{J}_{m}$ distinct de $(-x, y)$ est $\mathrm{J}_{m}$. 


\section{J. DÉSERTI}

Le centralisateur dans $\mathrm{J}_{0}$ d'un élément non trivial de $\mathrm{J}_{F}$ distinct de $(F(y) / x, y)$ est $\mathrm{J}_{F}$.

Le centralisateur dans $\mathrm{J}_{0}$ de $(-x, y)$ est $\left\{(a(y) x, y),(1 / b(y) x, y) \mid a, b \in \mathbb{C}(y)^{*}\right\}$.

Le centralisateur dans $\mathrm{J}_{0}$ de $(F(y) / x, y)$, où $F$ désigne un élément de $\mathbb{C}(y)^{*}$ qui n'est pas un carré, est :

$$
\left\{\left(\frac{a(y) x+F(y)}{x+a(y)}, y\right),\left(\frac{b(y) x-F(y)}{x-b(y)}, y\right),(-x, y) \mid a, b \in \mathbb{C}(y)\right\}
$$

La démonstration de ce lemme est purement calculatoire.

Proposition 3.3. Soit $H$ un sous-groupe abélien de $\mathrm{J}_{0}$. Alors $H$ est, à conjugaison près, un sousgroupe de $\mathrm{J}_{a}, \mathrm{~J}_{m}, \mathrm{~J}_{F}, \mathrm{I}_{B}$ ou $\mathrm{I}_{F}^{C}$. Les groupes $\mathrm{J}_{a}, \mathrm{~J}_{m}$ et $\mathrm{J}_{F}$ sont des sous-groupes abéliens maximaux de $\mathrm{J}$; tous sont maximaux dans $\mathrm{J}_{0}$.

\section{Démonstration.}

Commençons par montrer que les groupes $\mathrm{J}_{a}$, $\mathrm{J}_{m}$ et $\mathrm{J}_{F}$ (respectivement $\mathrm{I}_{B}$ et $\mathrm{I}_{F}^{C}$ ) sont maximaux dans $\mathrm{J}$ (respectivement $\mathrm{J}_{0}$ ). Soit $f$ dans le groupe de Jonquières qui commute à $\mathrm{J}_{a}$; en écrivant la commutation de $f$ avec $\left(x+y^{k}, y\right)$ pour tout $k \geqslant 0$, nous obtenons que $f$ appartient à $\mathrm{J}_{a}$.

Soit $f$ un élément du groupe de Jonquières commutant aux éléments de $\mathrm{J}_{m}$. Alors $f$ appartient à $\mathrm{J}_{m}$ : il suffit d'écrire, par exemple, la commutation de $f$ avec les transformations $(2 x, y)$ et $(y x, y)$. Ainsi $\mathrm{J}_{m}$ est maximal dans le groupe $\mathrm{J}$.

Chaque groupe $\mathrm{J}_{F}$ est maximal dans le groupe de Jonquières. En effet, soit $f$ un élément de $\mathrm{J} ; f$ commute aux éléments $\psi(a, F, 1, a ; y)$ de $\mathrm{J}_{F}$ si et seulement si les points fixes $(x, y)$ de $f$ sont donnés par $x^{2}=F(y)$ et :

$$
a^{2}(y) F(\pi(f))=a^{2}(\pi(f)) F(y)
$$

pour tout $a$ dans $\mathbb{C}(y)$. Ainsi les fonctions rationnelles $a^{2} / F$ sont toutes invariantes par $\pi(f)$ qui est donc nécessairement trivial. Ceci implique que $f$ appartient à $\mathrm{J}_{0}$; un calcul direct montre alors que $f$ est dans $\mathrm{J}_{F}$.

Le groupe $\mathrm{I}_{B}$ est un sous-groupe abélien maximal de $\mathrm{J}_{0}$. Ceci résulte du lemme 3.2 et du fait que $\mathrm{I}_{B}$ est le centralisateur dans $\mathrm{J}_{0}$ de $(1 / B(y) x, y)$.

De même, puisque les éléments $\psi(b, F, 1, b ; y)$ et $\psi(a,-F, a,-a ; y)$ commutent si et seulement si $b$ est nul, le lemme 3.2 assure que $\mathrm{I}_{F}^{C}$ est abélien maximal dans $\mathrm{J}_{0}$.

Montrons que $\mathrm{J}_{a}, \mathrm{~J}_{m}, \mathrm{~J}_{F}, \mathrm{I}_{B}$ et $\mathrm{I}_{F}^{C}$ sont, à conjugaison près, les seuls sous-groupes abéliens maximaux de $\mathrm{J}_{0} \simeq \mathrm{PGL}_{2}(\mathbb{C}(y))$. Fixons un élément non trivial $g=\psi(a, b, c, d ; y)$ d'un sous-groupe abélien maximal $H$ de $\mathrm{J}_{0}$. Quitte à faire un changement de coordonnées, nous pouvons supposer que $g$ s'écrit sous forme normale :

$$
\left(\frac{\alpha(y) x+\beta(y)}{\gamma(y) x+\alpha(y)}, y\right)
$$

(a) Lorsque $\alpha$ est nul, $g$ s'écrit $\psi(0, F, 1,0 ; y)$ où $F(y)=\beta(y) / \gamma(y)$.

Si $F$ n'est pas un carré, le lemme 3.2 assure qu'une transformation $h$ de $H$ qui commute à $g$ est de l'un des types suivants: $(-x, y), \psi(a, F, 1, a ; y)$ ou $\psi(a,-F, 1,-a ; y)$. Si $h=(-x, y)$, alors, toujours d'après le lemme 3.2 , le groupe $H$ coïncide avec $\mathrm{I}_{1 / F}$. Sinon suivant que $H$ contient un élément de la forme $\psi(C, F, 1, C ; y)$, avec $C \not \equiv 0$, ou non, nous obtenons $H=\mathrm{J}_{F}$ ou $H=\mathrm{I}_{F}^{C}$.

Si $F$ s'écrit $\tilde{F}^{2}$, alors $g$ est conjugué, via $\psi(-\tilde{F} ; \tilde{F}, 1,1 ; y)$, à $(-x, y)$. Nous obtenons alors les groupes $\mathrm{I}_{B}$ ou $\mathrm{J}_{m}$.

(b) Etudions le cas où $\alpha$ est non nul, i.e. $g$ est du type $\psi(1, b, c, 1 ; y)$.

Si $c$ est nul, alors $g$ appartient à $\mathrm{J}_{a}$ et le lemme 3.2 assure que $H=\mathrm{J}_{a}$. Si $c$ est non nul, alors $g$ s'écrit $\psi(1 / c, b / c, 1,1 / c ; y)$. Supposons que $b / c$ ne soit pas un carré ; $g$ est un élément de $\mathrm{J}_{b / c}$ et le 


\section{Automorphismes du Groupe De Cremona}

lemme 3.2 assure que $H$ coïncide avec $\mathrm{J}_{b / c}$. Si $b / c$ est un carré, alors $g$ est, à conjugaison près, dans $\mathrm{J}_{m}$; d'après le lemme 3.2 nous avons, à conjugaison près, $H=\mathrm{J}_{m}$ ou $H=\mathrm{I}_{B}$.

\subsection{Propriétés de $\mathbf{J}_{a}$}

Voici deux propriétés de $\mathrm{J}_{a}$ qui vont nous permettre de le distinguer des autres sous-groupes abéliens maximaux non dénombrables de $\operatorname{Bir}\left(\mathbb{P}^{2}(\mathbb{C})\right)$ :

- $\mathrm{J}_{a}$ ne contient pas d'élément de torsion ;

- le groupe non virtuellement résoluble $\left\{(a(y) x+b(y), \nu(y)) \mid a \in \mathbb{C}(y)^{*}, b \in \mathbb{C}(y), \nu \in \operatorname{PGL}(2, \mathbb{C})\right\}$ agit par conjugaison sur $\mathrm{J}_{a}$.

Remarque 3.4. Les groupes $\mathrm{J}_{m}$ et $\mathrm{J}_{F}$ ne sont pas isomorphes à $\mathrm{J}_{a}$ car ils contiennent des éléments de torsion.

\section{Sous-groupes abéliens maximaux non dénombrables de J}

Commençons par remarquer le fait suivant :

LEMme 4.1. Les groupes $\mathrm{J}_{a}$ et $\mathrm{T}$ ne sont pas conjugués.

Démonstration. Un calcul élémentaire montre que tout sous-groupe de $\operatorname{Bir}\left(\mathbb{P}^{2}(\mathbb{C})\right)$ agissant par conjugaison sur $\mathrm{T}$ est contenu dans le groupe affine $\{(a x+b y+c, d x+e y+f) \mid a, b, c, d, e, f \in \mathbb{C}\}$. Supposons que $\mathrm{J}_{a}$ et $\mathrm{T}$ soient isomorphes, alors $\{(x, \nu(y)) \mid \nu \in \operatorname{PGL}(2, \mathbb{C})\} \simeq \operatorname{PSL}(2, \mathbb{C})$ s'injecte dans $\{(a x+b y+c, d x+e y+f) \mid a, b, c, d, e, f \in \mathbb{C}\} ; \operatorname{comme} \operatorname{PSL}(2, \mathbb{C})$ est simple, nous en déduisons une injection $j$ de $\operatorname{PSL}(2, \mathbb{C})$ dans $H=\{(a x+b y+c, d x+e y+f) \mid a, b, c, d, e, f \in \mathbb{C}, a e-b d=1\}$. Notons $q: H \rightarrow \operatorname{SL}(2, \mathbb{C})$ l'application 'partie linéaire' et $p: \operatorname{SL}(2, \mathbb{C}) \rightarrow \operatorname{PSL}(2, \mathbb{C})$ la projection canonique. Si $p \circ q$ était trivial, alors $j(\operatorname{PSL}(2, \mathbb{C}))$ serait métabélien ce qui est impossible donc $p \circ q$ n'est pas trivial ; par simplicité de $\operatorname{PSL}(2, \mathbb{C})$, l'application $p \circ q \circ j$ est injective. Il s'en suit que $q \circ j: \operatorname{PSL}(2, \mathbb{C}) \rightarrow \mathrm{SL}(2, \mathbb{C})$ aussi, ce qui est impossible.

ThÉorème 4.2. Soit G un sous-groupe abélien maximal non dénombrable du groupe de Jonquières. Le groupe $\mathrm{G}$ vérifie l'une des propriétés suivantes :

- G contient un élément de torsion ;

- tout sous-groupe de $\operatorname{Bir}\left(\mathbb{P}^{2}(\mathbb{C})\right)$ agissant par conjugaison sur $\mathrm{G}$ est virtuellement résoluble ;

- G est conjugué à $\mathrm{J}_{a}$;

- G est conjugué à T.

Nous déduisons des théorèmes $2.3,4.2$, des remarques $3.4,4.1$ et des propriétés de $\mathrm{J}_{a}$ (§ 3.3 ) le : Corollaire 4.3. Soit G un sous-groupe abélien maximal non dénombrable du groupe de Jonquières. Si G est isomorphe à $\mathrm{J}_{a}$, alors $\mathrm{G}$ est conjugué à $\mathrm{J}_{a}$.

La suite de cette partie est consacrée à la démonstration du théorème 4.2. Remarquons que si $\pi(\mathrm{G})$ est trivial, le théorème 4.2 est une conséquence de la proposition 3.3.

Les deux énoncés qui suivent sont des résultats techniques auxquels nous nous référerons souvent.

LEMme 4.4. Soit G un sous-groupe abélien maximal non dénombrable du groupe de Jonquières tel que $\mathrm{G}_{0}$ soit un sous-groupe non trivial de $\mathrm{J}_{m}$. Alors $\mathrm{G}$ contient un élément de torsion.

Démonstration. Soient $g=(a(y) x, y)$ un élément non trivial de $\mathrm{G}_{0}$ et $f=\left(f_{1}, f_{2}\right)$ dans $\mathrm{G}$. La condition de commutation de $f$ et $g$ implique que $f_{1}$ est de l'un des deux types $b(y) x$ ou $1 / b(y) x$; ceci permet de remarquer que $(-x, y)$ commute à $f$. Par maximalité l'involution $(-x, y)$ appartient à $G$. 


\section{J. DÉSERTI}

Lemme 4.5. Soit $\mathrm{G}$ un sous-groupe abélien maximal non dénombrable de $\mathrm{J}$ tel que $\pi(\mathrm{G})$ soit infini et $\mathrm{G}_{0}$ un sous-groupe non trivial de $\mathrm{J}_{a}$. Après conjugaison par un élément de $\mathrm{J}_{m}$ nous pouvons supposer que :

(i) $(x+1, y)$ appartient à $\mathrm{G}_{0}$;

(ii) G est contenu dans $\{(x+b(y), \nu(y)) \mid b \in \mathbb{C}(y), \nu \in \pi(\mathrm{G})\}$;

(iii) $\mathrm{G}_{0}=\left\{(x+a(y), y) \mid a \in \mathbb{C}(y)^{\pi(\mathrm{G})}\right\}$ où $\mathbb{C}(y)^{\pi(\mathrm{G})}$ désigne le corps des fonctions rationnelles invariantes par $\pi(\mathrm{G})$.

Démonstration. (i) Par hypothèse $\mathrm{G}_{0}$ contient un élément du type $(x+b(y), y)$ avec $b$ non nul qui, à conjugaison près par $(b(y) x, y)$, s'écrit $(x+1, y)$.

(ii) Une transformation birationnelle qui commute à $(x+1, y)$ s'écrit $(x+b(y), \nu(y))$ où $b$ désigne un élément de $\mathbb{C}(y)$ et $\nu$ un élément de $\operatorname{PGL}(2, \mathbb{C})$.

(iii) Soient $(x+a(y), y)$ un élément de $\mathrm{G}_{0}$ et $(x+b(y), \nu(y))$ un élément de $\mathrm{G} \backslash \mathrm{G}_{0}$; la commutation de ces deux transformations conduit à l'égalité $a(y)=a(\nu(y))$.

\subsection{Cas $\pi(\mathrm{G})$ fini non trivial}

Ici nous pouvons supposer, à conjugaison près, que $\pi(\mathrm{G})=\langle-y, 1 / y\rangle$ ou que $\pi(\mathrm{G})=\langle\alpha y\rangle$ avec $\alpha$ racine $q$-ième de l'unité.

Lemme 4.6. Soit G un sous-groupe abélien maximal non dénombrable du groupe de Jonquières tel que $\pi(\mathrm{G})$ soit fini non trivial. Alors $\mathrm{G}$ contient un élément de torsion.

Démonstration. $\mathrm{Si}_{0}$ est un sous-groupe de $\mathrm{J}_{m}$, le lemme 4.4 permet de conclure.

Supposons que $G_{0}$ soit un sous-groupe de $J_{a}$ et que $\pi(G)$ soit engendré par un élément d'ordre fini.

En reprenant les calculs de la démonstration de la proposition 2.5, où nous substituons les séries de Laurent aux développements en séries entières, nous obtenons que G contient au moins un élément de torsion.

Considérons le cas où $\mathrm{G}_{0}$ est un sous-groupe de $\mathrm{J}_{F}$ et $\pi(\mathrm{G})$ est engendré par $\alpha y$, un élément d'ordre fini $q$.

Soient $f$ dans $\mathrm{G}$ tel que $\pi(f)=\alpha y$ et $g=\psi(a, F, 1, a ; y)$ dans $\mathrm{G}_{0}$. La commutation de $f$ et $g$ se traduit par $a^{2}(\alpha y) F(y)=a^{2}(y) F(\alpha y)$. En particulier, si $\psi(\tilde{a}, F, 1, \tilde{a} ; y)$ est un élément de $\mathrm{G}_{0}$, non trivial et distinct de $g$, nous obtenons l'égalité $a^{2}(y) \tilde{a}^{2}(\alpha y)=a^{2}(\alpha y) \tilde{a}^{2}(y)$ qui conduit à $\tilde{a}(y)=\ell\left(y^{q}\right) a(y)$ où $\ell$ appartient à $\mathbb{C}(y)$.

Un calcul direct montre que $F$ est une fonction de $y^{q}$; de plus $g$ et $f$ s'écrivent respectivement à conjugaison près $\psi\left(\ell\left(y^{q}\right), F\left(y^{q}\right), 1, \ell\left(y^{q}\right) ; y\right)$ et $\psi\left(b, c F\left(y^{q}\right), c, b ; \alpha y\right)$. Autrement dit

$$
\mathrm{G}_{0}=\left\{\left(\frac{\ell\left(y^{q}\right) x+F\left(y^{q}\right)}{x+\ell\left(y^{q}\right)}, y\right) \mid \ell \in \mathbb{C}(y)\right\}
$$

et $\mathrm{G}$ contient au moins un élément de torsion : $\left(F\left(y^{q}\right) / x, y\right)$.

Supposons que $\mathrm{G}_{0}$ soit un sous-groupe de $\mathrm{J}_{a}$ et que $\pi(\mathrm{G})$ soit le groupe à quatre éléments $\langle-y, 1 / y\rangle$.

Adoptons les notations suivantes :

$$
H_{1}=\langle-y\rangle, \quad h_{1}(y)=-y, \quad \eta_{1}(y)=y^{2}, \quad H_{2}=\left\langle\frac{1}{y}\right\rangle, \quad h_{2}(y)=\frac{1}{y} \quad \text { et } \quad \eta_{2}(y)=y+\frac{1}{y} .
$$

Les fonctions $\eta_{i}$ sont invariantes sous l'action des $H_{i}$; toute fonction invariante par le groupe $\langle-y, 1 / y\rangle$ se factorise dans $y^{2}+1 / y^{2}$. 


\section{Automorphismes Du Groupe De Cremona}

En écrivant qu'un élément de $\mathrm{G}_{0}$ et un élément de $\pi^{-1}\left(H_{i}\right) \backslash \mathrm{G}_{0}$ commutent, puis que deux transformations de $\pi^{-1}\left(H_{i}\right) \backslash \mathrm{G}_{0}$ commutent, nous obtenons :

$$
\pi^{-1}\left(H_{i}\right)=\left\{\left(x+c\left(\eta_{i}(y)\right), y\right),\left(x+b_{i}(y)+r\left(\eta_{i}(y)\right), h_{i}(y)\right) \mid c \in \Theta_{i}, r \in \tilde{\Theta}_{i}\right\},
$$

où $\Theta_{i}, \tilde{\Theta}_{i}$ désignent deux sous-groupes additifs de $\mathbb{C}(y)$ et $b_{i}$ un élément fixé de $\mathbb{C}(y)$.

Par maximalité de $\mathrm{G}$ et puisque $\mathrm{G}_{0}=\pi^{-1}\left(H_{1} \cap H_{2}\right)$, nous avons :

$$
\mathrm{G}_{0}=\left\{\left(x+c\left(y^{2}+1 / y^{2}\right), y\right) \mid c \in \mathbb{C}(y)\right\}
$$

en particulier $\Theta_{i}=\mathbb{C}(y)$.

Soit $f=\left(x+b_{1}(y)+r\left(y^{2}\right),-y\right)$ dans $\pi^{-1}\left(H_{1}\right) \backslash \mathrm{G}_{0}$; écrivons $b_{1}$ sous la forme $\tilde{b}_{1}\left(y^{2}\right)+y \bar{b}_{1}\left(y^{2}\right)$. Quitte à conjuguer $f$ par :

$$
\left(x+y \bar{b}_{1}\left(y^{2}\right) / 2, y\right)
$$

nous pouvons supposer que $f$ est du type $\left(x+e\left(y^{2}\right),-y\right)$; une telle conjugaison n'a pas d'effet sur le noyau et laisse $\pi^{-1}\left(H_{2}\right)$ invariant. La transformation $f^{2}=\left(x+2 e\left(y^{2}\right), y\right)$ est dans $\mathrm{G}_{0}$ donc $e$ se factorise dans $y^{2}+1 / y^{2}$. Ainsi

$$
(x,-y)=\left(x-2 e\left(y^{2}+1 / y^{2}\right), y\right) \circ f
$$

appartient à $\pi^{-1}\left(H_{1}\right)$ : le groupe $\mathrm{G}$ possède donc au moins un élément de torsion.

Finalement étudions le cas où $\mathrm{G}_{0}$ est un sous-groupe de $\mathrm{J}_{F}$ et $\pi(\mathrm{G})=\langle-y, 1 / y\rangle$.

Un élément de $\mathrm{G}_{0}$ et un élément de $\pi^{-1}\left(H_{i}\right) \backslash \mathrm{G}_{0}$ commutent si et seulement s'ils s'écrivent :

$$
\psi\left(\ell\left(\eta_{i}(y)\right), F\left(\eta_{i}(y)\right), 1, \ell\left(\eta_{i}(y)\right) ; y\right) \quad \text { et } \quad \psi\left(a, b(y) F\left(\eta_{i}(y)\right), b, a ; h_{i}(y)\right) .
$$

Ceci étant valable pour $i=1,2$, nous en déduisons que $F$ et $\ell$ se factorisent dans $y^{2}+1 / y^{2}$. Par maximalité de G, nous obtenons :

$$
\mathrm{G}_{0}=\left\{\left(\frac{\ell\left(y^{2}+1 / y^{2}\right) x+F\left(y^{2}+1 / y^{2}\right)}{x+\ell\left(y^{2}+1 / y^{2}\right)}, y\right) \mid \ell \in \mathbb{C}(y)\right\} ;
$$

le groupe $\mathrm{G}$ contient donc l'élément de torsion $\left(F\left(y^{2}+1 / y^{2}\right) / x, y\right)$.

\subsection{Cas $\pi(\mathrm{G})$ infini}

Dans ce cas, nous pouvons supposer que $\mathrm{G}_{0}$ est un sous-groupe de $\mathrm{J}_{a}, \mathrm{~J}_{m}, \mathrm{I}_{B}$ ou $\mathrm{I}_{F}^{C}$. En effet, nous avons le :

Lemme 4.7. Soit G un sous-groupe abélien maximal non dénombrable du groupe de Jonquières. Si $\mathrm{G}_{0} \cap \mathrm{J}_{F}$ est non trivial, alors $\pi(\mathrm{G})$ est fini ou $\mathrm{G}$ contient un élément de torsion.

Démonstration. Supposons qu'il existe dans $\mathrm{J}_{F} \cap \mathrm{G}_{0}$ un élément $g$ de la forme

$$
((a(y) x+F(y)) /(x+a(y)), y)
$$

avec $a$ non nul. Comme $F$ n'est pas un carré, la fonction $F / a^{2}$ n'est pas constante. Soit $f=\left(f_{1}, \nu\right)$ dans G. La commutation de $f$ et $g$ entraîne que les matrices

$$
\left(\begin{array}{ll}
a & F \\
1 & a
\end{array}\right) \text { et }\left(\begin{array}{cc}
a & F \\
1 & a
\end{array}\right) \circ \nu
$$

de $\operatorname{PGL}(2, \mathbb{C}(y))$ sont conjuguées. Puisque det/(trace $)^{2}$ est un invariant de conjugaison, un calcul rapide conduit à :

$$
\frac{F}{a^{2}} \circ \nu=\frac{F}{a^{2}}, \quad \forall \nu \in \pi(\mathrm{G}) .
$$

Comme $F$ n'est pas un carré, la fonction $F / a^{2}$ n'est pas constante et le groupe $\pi(\mathrm{G})$ est fini.

Si $\mathrm{J}_{F} \cap \mathrm{G}_{0}$ est réduit à $\langle(F(y) / x, y)\rangle$, alors $\mathrm{G}$ contient un élément de torsion. 


\section{J. DÉSERTI}

Rappelons que si $\mathrm{G}_{0}$ est un sous-groupe non trivial de $\mathrm{J}_{m}$, le groupe $\mathrm{G}$ contient un élément de torsion (lemme 4.4).

Seules restent à examiner les deux éventualités suivantes : $\mathrm{G}_{0}$ est un sous-groupe non trivial de $\mathrm{J}_{a}$ ou $\mathrm{G}_{0}$ est un sous-groupe de $\mathrm{I}_{B}$ ou $\mathrm{I}_{F}^{C}$.

\subsubsection{Cas $\mathrm{G}_{0}$ sous-groupe non trivial de $\mathrm{J}_{a}$.}

LEMme 4.8. Soit $f$ une fonction rationnelle non nulle telle que pour a et $\lambda$ deux complexes non nuls $f(x+a, y)=\lambda f(x, y) ;$ alors $\lambda$ vaut 1 .

Démonstration. En dérivant $f(x+a, y)=\lambda f(x, y)$ par rapport à $x$, nous obtenons l'égalité $(\partial f / \partial x)(x+a, y)=\lambda(\partial f / \partial x)(x, y)$. Remarquons que $(\partial f / \partial x) / f$ est invariant par $(x+a, y)$ donc ne dépend pas de $x$. Comme $f$ est rationnelle $f$ ne dépend que de $y$ et nécessairement $\lambda$ vaut 1 .

ThÉorème 4.9. Soit G un sous-groupe abélien maximal non dénombrable du groupe de Jonquières. Si $\pi(\mathrm{G})$ est infini et $\mathrm{G}_{0}$ un sous-groupe non trivial de $\mathrm{J}_{a}$, alors à conjugaison près :

(i) $\mathrm{G}_{0}=\{(x+a, y) \mid a \in \mathbb{C}\}$;

(ii) tout sous-groupe agissant par conjugaison sur G est virtuellement résoluble ;

(iii) si $\pi(\mathrm{G})$ est un sous-groupe non dénombrable de translations, alors $\mathrm{G}$ est conjugué à T.

Démonstration. Le (i) est une conséquence directe du lemme 4.5 ; en effet, une fonction rationnelle $a(y)$ invariante par une infinité d'éléments $\nu$ de $\operatorname{PGL}(2, \mathbb{C})$ est constante. Dans la suite nous allons montrer les assertions (ii) et (iii) en raisonnant suivant la nature de $\pi(\mathrm{G})$.

Rappelons que les éléments de $\mathrm{G} \backslash \mathrm{G}_{0}$ sont de la forme $(x+b(y), \nu(y))$ (lemme 4.5). Puisque $\mathrm{G}_{0}=\{(x+a, y) \mid a \in \mathbb{C}\}$, chaque élément $\nu$ de $\pi(\mathrm{G})$ a un unique antécédent $(x+b(y), \nu(y))$ tel que le terme constant du développement en série de Laurent de $b$ à l'origine soit nul ; nous le noterons $\left(x+b_{\nu}(y), \nu(y)\right)$.

Commençons par étudier le cas où $\pi(\mathrm{G})$ est un groupe d'homothéties. Soient $H$ un sous-groupe de $\operatorname{Bir}\left(\mathbb{P}^{2}(\mathbb{C})\right)$ agissant par conjugaison sur $G$ et $h=\left(h_{1}, h_{2}\right)$ un élément de $H$. S'il existait $g=(x+a, y)$ dans $\mathrm{G}_{0}$ tel que $h g h^{-1}$ s'écrive $(x+b(y), \gamma y)$ avec $\gamma \neq 1$, alors $h_{2}$ vérifierait $h_{2}(x+a, y)=\gamma h_{2}(x, y)$ ce qui, d'après le lemme 4.8 , est impossible. Ainsi pour tout $g$ dans $\mathrm{G}_{0}$ la transformation $h g h^{-1}$ appartient à $\mathrm{G}_{0}$; par conséquent pour tout $f$ dans $\mathrm{G} \backslash \mathrm{G}_{0}$ l'élément $h f h^{-1}$ est dans $\mathrm{G} \backslash \mathrm{G}_{0}$. Il s'en suit que $h_{1}(x+1, y)=h_{1}(x, y)+\xi$ pour un certain $\xi$ et $h_{2}(x+1, y)=h_{2}(x, y)$; en particulier, $h_{1}$ est de la forme $\alpha x+c(y)$ et $h_{2}$ est une fonction de $y$. Soit $f=\left(f_{1}, \beta y\right)$ un élément de $\mathrm{G} \backslash \mathrm{G}_{0}$; comme $h f^{-1}$ appartient à $\mathrm{G} \backslash \mathrm{G}_{0}$, la seconde composante de $h$ est du type $\mu y^{ \pm 1}$. Finalement $h$ s'écrit $\left(\alpha x+c(y), \mu y^{ \pm 1}\right)$ et tout sous-groupe de $\operatorname{Bir}\left(\mathbb{P}^{2}(\mathbb{C})\right)$ agissant par conjugaison sur $\mathrm{G}$ est virtuellement résoluble.

Supposons que $\pi(\mathrm{G})$ soit un groupe dénombrable de translations. Soit $f$ une transformation birationnelle agissant par conjugaison sur $\mathrm{G}$; en considérant la restriction de l'action par conjugaison de $f$ à $\mathrm{G}_{0}$, nous constatons qu'une infinité non dénombrable d'éléments du type $(x+a, y)$ est envoyé sur des éléments du même type. Un calcul direct montre alors que $f$ s'écrit $(\alpha x+a(y), \beta y+\gamma)$, où $\alpha, \beta$ appartiennent à $\mathbb{C}^{*}, \gamma$ à $\mathbb{C}$ et $a$ à $\mathbb{C}(y) ;$ autrement dit tout sous-groupe de $\operatorname{Bir}\left(\mathbb{P}^{2}(\mathbb{C})\right)$ agissant par conjugaison sur $\mathrm{G}$ est résoluble.

Considérons le cas où $\pi(\mathrm{G})$ est un sous-groupe non dénombrable de translations. Il existe un entier $n$ tel que $\mathrm{G}_{n}=\{(x+C(y), y+\alpha) \in \mathrm{G} \mid \operatorname{deg} C=n\}$ ne soit pas dénombrable. Notons $E_{n}^{0}$ l'ensemble des éléments $C$ de $\mathbb{C}(y)$ de degré $n$ dont le terme constant $c_{0}(C)$ dans le développement en série de Laurent est nul :

$$
E_{n}^{0}=\left\{C \in \mathbb{C}(y) \mid \operatorname{deg} C=n, c_{0}(C)=0\right\}
$$




\section{Automorphismes Du Groupe De Cremona}

L'ensemble :

$$
\Omega_{n}:=\left\{(C, \beta) \in E_{n}^{0} \times \mathbb{C} \mid C_{\alpha}(y+\beta)-C_{\alpha}(y)=C(y+\alpha)-C(y), \forall y+\alpha \in \pi(\mathrm{G})\right\}
$$

est une sous-variété algébrique de $E_{n}^{0} \times \mathbb{C}$. La normalisation par $c_{0}=0$ et le fait que $\mathrm{G}_{n}$ soit non dénombrable impliquent que $\Omega_{n}$ est de dimension un. Notons $p$ la projection de $\mathbb{C}(y) \times \mathbb{C}$ sur $\mathbb{C}$. L'image de $\Omega_{n}$ par $p$ est un sous-ensemble constructible de $\mathbb{C}$ qui contient un ensemble non dénombrable ; donc $p\left(\Omega_{n}\right)=\mathbb{C} \backslash\left\{\beta_{1}, \ldots, \beta_{k}\right\}$ et $p$ est injective sur $\Omega_{n}$. L'ensemble $\Omega_{n}$ est inclus dans G. En effet, une transformation $f$ de $\mathrm{G}$ commute aux éléments de $\mathrm{G}_{n}$ et $\mathrm{G}_{n}$ est Zariski dense dans $\Omega_{n}$; par suite $f$ commute aux transformations $\left(x+C_{\beta}(y), y+\beta\right)$ où $(C, \beta)$ appartient à $\Omega_{n}$. En particulier, comme $\pi(\mathrm{G})$ est un groupe, $\pi(\mathrm{G})$ est isomorphe à $\mathbb{C}$. L'application $\beta \mapsto\left(C_{\beta}, \beta\right)$, définie sur $\mathbb{C} \backslash\left\{\beta_{1}, \ldots, \beta_{k}\right\}$, est une paramétrisation injective de $\Omega_{n}$ donc holomorphe sur $\mathbb{C} \backslash\left\{\beta_{1}, \ldots, \beta_{k}\right\}$; il s'en suit que $\beta \mapsto C_{\beta}$ est rationnelle sur $\mathbb{C}$ (argument de compactification). Notons $C(\alpha, y)=C_{\alpha}(y)$. Dérivons l'égalité :

$$
C(\alpha, y)-C(\alpha, y+\beta)=C(\beta, y)-C(\beta, y+\alpha)
$$

par rapport à $\alpha$ et $\beta$, nous obtenons :

$$
\frac{\partial^{2} C}{\partial \alpha \partial y}(\alpha, y+\beta)=\frac{\partial^{2} C}{\partial \alpha \partial y}(\beta, y+\alpha)
$$

Posons $A=\partial^{2} C / \partial \alpha \partial y$. Dérivons l'égalité (3) en $\alpha$ et en $y$, alors $(\partial A / \partial \alpha)(\alpha, y+\beta)=(\partial A / \partial y)$ $(\alpha, y+\beta)$; autrement dit $A$ annule le champ $\partial / \partial \alpha-\partial / \partial y$. Ainsi $\partial^{2} C / \partial \alpha \partial y$ est une fonction de $y+\alpha$. Notons que $C_{\alpha}(y)$ est rationnelle en $\alpha$ et $y$; il en est donc de même pour $\partial^{2} C / \partial \alpha \partial y$. Par conséquent nous avons :

$$
C_{\alpha}(y)=L(y+\alpha)+l_{1}(\alpha)+l_{2}(y)
$$

où $L, l_{1}$ et $l_{2}$ sont rationnelles. La condition de commutation (égalité $(2)$ ) conduit à $l_{2}(t)=-L(t)$. Il s'en suit que $\left(x+C_{\alpha}(y), y+\alpha\right)$ s'écrit $\left(x+L(y+\alpha)-L(y)+l_{1}(\alpha), y+\alpha\right)$. Or $(x+L(y+\alpha)-$ $\left.L(y)+l_{1}(\alpha), y+\alpha\right)$ est conjugué à $\left(x+l_{1}(\alpha), y+\alpha\right)$ via $(x+L(y), y)$.

Cette conjugaison laisse $\mathrm{G}_{0}$ invariant donc $\mathrm{G}$ est, à conjugaison près, un sous-groupe de $\mathrm{T}$; par maximalité cette inclusion est une égalité.

4.2.2 $\mathrm{G}_{0}$ sous-groupe de $\mathrm{I}_{B}$ ou $\mathrm{I}_{F}^{C}$. Remarquons que si $\mathrm{G}_{0}$ est conjugué à un sous-groupe non trivial de $\mathrm{I}_{B}$ ou de $\mathrm{I}_{F}^{C}$, le groupe $\mathrm{G}$ contient un élément de torsion car les groupes $\mathrm{I}_{B}$ et $\mathrm{I}_{F}^{C}$ sont engendrés par deux involutions. Reste donc à étudier le cas où $\mathrm{G}_{0}$ est trivial ; nécessairement $\pi(\mathrm{G})$ est un sous-groupe non dénombrable d'homothéties ou de translations.

Introduisons la variété algébrique $K_{n}$ définie par :

$$
K_{n}=\left\{(f, \nu) \in \operatorname{PGL}(2, \mathbb{C}(y)) \rtimes \operatorname{PGL}(2, \mathbb{C}) \mid \operatorname{deg} f \leqslant n, \operatorname{deg} f^{-1} \leqslant n\right\} .
$$

Proposition 4.10. Soit G un sous-groupe abélien non dénombrable du groupe de Jonquières. Si $\mathrm{G}_{0}$ est trivial, alors $\mathrm{G}$ est algébrique, i.e. les éléments de $\mathrm{G}$ sont de degré borné et $\mathrm{G}$ est une sous-variété algébrique de $K_{n}$.

Démonstration. Elle procède du même principe qu'au théorème 4.9. Il existe un entier $n$ tel que $\mathrm{G}_{n}=\left\{(f, \nu) \in \mathrm{G} \cap K_{n}\right\}$ ne soit pas dénombrable. Notons $\overline{\mathrm{G}_{n}}$ l'adhérence de Zariski de $\mathrm{G}_{n}$ dans $K_{n}$. Soit $h$ une transformation de $\mathrm{G}$; elle commute aux éléments de $\mathrm{G}_{n}$. Comme $\mathrm{G}_{n}$ est Zariski dense dans $\overline{\mathrm{G}_{n}}$, la transformation $h$ commute à celles de $\overline{\mathrm{G}_{n}}$; ainsi $\overline{\mathrm{G}_{n}}$ est contenu dans G. Soit $p$ la projection de $\operatorname{PGL}(2, \mathbb{C}(y)) \rtimes \operatorname{PGL}(2, \mathbb{C})$ sur $\operatorname{PGL}(2, \mathbb{C})$. Quitte à identifier $y+\alpha$ (respectivement $\alpha y$ ) avec $\alpha$, l'image de $\overline{\mathrm{G}_{n}}$ par $p$ est un sous-ensemble constructible non dénombrable de $\mathbb{C}$ (respectivement $\left.\mathbb{C}^{*}\right)$ donc $p\left(\overline{\mathrm{G}_{n}}\right)=\mathbb{C} \backslash\left\{\alpha_{1}, \ldots, \alpha_{s}\right\}$ (respectivement $p\left(\overline{\mathrm{G}_{n}}\right)=\mathbb{C}^{*} \backslash\left\{\alpha_{1}, \ldots, \alpha_{s}\right\}$ ). Le groupe $p(\mathrm{G}$ ) est un sous-groupe de $\mathbb{C}$ (respectivement $\mathbb{C}^{*}$ ) contenant un ouvert donc $p(\mathrm{G})$ est isomorphe à $\mathbb{C}$ 


\section{J. DÉSERTI}

(respectivement $\mathbb{C}^{*}$ ). Puisque $p$ est injective sur $\mathrm{G}$, elle l'est sur $\overline{\mathrm{G}_{n}}$ qui, par suite, est de dimension un. Il s'en suit que $\mathrm{G}=\overline{\mathrm{G}_{n}} \cup\left\{g^{-1}\left(\alpha_{1}\right), \ldots, g^{-1}\left(\alpha_{s}\right)\right\}$, où $g^{-1}\left(\alpha_{i}\right)$ désigne l'élément de $\mathrm{G}$ tel que $\underline{p(g)}=y+\alpha_{i}$ (respectivement $p(g)=\alpha_{i} y$ ), est algébrique. En fait, a posteriori les $g^{-1}\left(\alpha_{i}\right)$ sont dans

Démonstration du théorème 4.2. Commençons par supposer que $\mathrm{G}_{0}$ n'est pas trivial. La proposition 3.3 et les propriétés de $\mathrm{J}_{a}(\S 3.3)$ assurent que lorsque $\pi(\mathrm{G})$ est trivial, G est isomorphe à $\mathrm{J}_{a}$ si et seulement si $G$ est conjugué à $\mathrm{J}_{a}$. D'après les lemmes 4.4 et 4.6 , si $\mathrm{G}_{0}$ est contenu dans $\mathrm{J}_{m}$ ou si $\pi(G)$ est fini et non trivial, alors $G$ contient un élément de torsion. Si $\pi(G)$ est infini, il suffit de considérer le cas où $\mathrm{G}_{0}$ est un sous-groupe de $\mathrm{J}_{a}$ (lemme 4.7) ; alors $\mathrm{G}$ satisfait l'une des propriétés suivantes (théorème 4.9):

- tout sous-groupe agissant par conjugaison sur G est virtuellement résoluble ;

- G est conjugué à T.

Enfin si $\mathrm{G}_{0}$ est trivial, le groupe $\mathrm{G}$ est algébrique (proposition 4.10 ) ; l'action de $\mathrm{G}$ sur $\mathbb{P}^{2}(\mathbb{C})$ étant clairement algébrique, $\mathrm{G}$ est, à conjugaison près, un sous-groupe du groupe des automorphismes d'une surface minimale (voir [Enr93, Ume82]). Le corollaire 2.6 assure alors que, par maximalité, $\mathrm{G}$ est conjugué à $\mathrm{T}$ ou contient un élément de torsion.

\section{Classification des automorphismes}

\subsection{Groupes de translations}

Rappelons que $\varphi$ désigne un automorphisme du groupe de Cremona. D'après le corollaire 4.3, nous pouvons supposer, quitte à faire une conjugaison, que $\mathrm{J}_{a}$ est invariant par $\varphi$ et que $\varphi(x+1, y)=$ $(x+1, y)$.

Supposons qu'à une certaine étape de la preuve, nous ayons montré que $\varphi$ fixe une famille $\left\{H_{i}\right\}_{i}$ de groupes. La conjugaison par une transformation birationnelle $f$ est dite permise si $f H_{i} f^{-1}=H_{i}$ pour tout $i$.

Pour tout couple $(\alpha, \beta)$ de $\mathbb{C}^{2}$, notons $t_{\alpha, \beta}$ l'application $(x+\alpha, y+\beta)$. Tout élément $t_{\alpha, \beta} \mathrm{du}$ groupe des translations s'écrit $t_{\alpha, 0} t_{0, \beta}$; introduisons donc les groupes $\mathrm{T}_{1}$ et $\mathrm{T}_{2}$ définis par $\mathrm{T}_{1}=$ $\left\{t_{\alpha, 0} \mid \alpha \in \mathbb{C}\right\}$ et $\mathrm{T}_{2}=\left\{t_{0, \alpha} \mid \alpha \in \mathbb{C}\right\}$.

Proposition 5.1. Soit $\varphi$ un automorphisme de $\operatorname{Bir}\left(\mathbb{P}^{2}(\mathbb{C})\right)$ qui fixe le groupe $\mathrm{J}_{a}$ et $(x+1, y)$. Alors, à conjugaison permise près, $\varphi$ préserve les groupes $\mathrm{T}_{i}$ et $\varphi\left(\left\{(x, \alpha y) \mid \alpha \in \mathbb{C}^{*}\right\}\right)$ est un sous-groupe de $\{(x+b(y), \nu(y)) \mid b \in \mathbb{C}(y), \nu \in \operatorname{PGL}(2, \mathbb{C})\}$.

Démonstration. Le groupe $\mathrm{T}$ est un sous-groupe abélien maximal non dénombrable $\operatorname{de} \operatorname{Bir}\left(\mathbb{P}^{2}(\mathbb{C})\right)$ donc $\varphi(\mathrm{T})$ aussi.

L'image de $\mathrm{T}_{2}$ par $\varphi$ est un sous-groupe de $\{(x+b(y), \nu(y)) \mid b \in \mathbb{C}(y), \nu \in \operatorname{PGL}(2, \mathbb{C})\}$. En effet tout élément de $\varphi\left(\mathrm{T}_{2}\right)$ agit trivialement par conjugaison sur $\varphi\left(\mathrm{T}_{1}\right)$, en particulier sur $t_{1,0}$. Ainsi pour tout $\left(h_{1}, h_{2}\right)$ dans $\varphi\left(\mathrm{T}_{2}\right)$ nous avons $h_{1}(x+1, y)=h_{1}(x, y)+1$ et $h_{2}(x+1, y)=h_{2}(x, y)$ ce qui montre que $h_{2}$ ne dépend pas de $x$ et que $h_{1}$ est de la forme $x+b(y)$.

Comme $\mathrm{T}_{1}$ est un sous-groupe de $\mathrm{J}_{a}$ et commute à $\mathrm{T}_{2}$, l'image de $\mathrm{T}_{1}$ par $\varphi$ est contenue dans $\mathrm{T}_{1}$.

Le groupe $\mathrm{D}_{2}=\left\{(x, \alpha y) \mid \alpha \in \mathbb{C}^{*}\right\}$ agit trivialement par conjugaison sur $\mathrm{T}_{1}$ donc $\varphi\left(\mathrm{D}_{2}\right)$ est un sous-groupe de $\{(x+b(y), \nu(y)) \mid b \in \mathbb{C}(y), \nu \in \operatorname{PGL}(2, \mathbb{C})\}$.

Soit $g$ un élément de $\varphi\left(\mathrm{T}_{2}\right) \cap \mathrm{T}_{1}$; il s'écrit $(x+a, y)$. Pour toute transformation $(x+b(y), \nu(y))$ de $\varphi\left(\mathrm{D}_{2}\right)$ nous avons $g(x+b(y), \nu(y))=(x+b(y), \nu(y)) g$. Le groupe $\mathrm{D}_{2}$ agissant sans point fixe sur $\mathrm{T}_{1}$, le complexe $a$ est nul. Ainsi $\varphi\left(\mathrm{T}_{2}\right) \cap \mathrm{T}_{1}$ est trivial. Puisque $\mathrm{T}$ est maximal et $\varphi\left(\mathrm{T}_{1}\right)$ est inclus dans $\mathrm{T}_{1}$, nous avons $\varphi\left(\mathrm{T}_{1}\right)=\mathrm{T}_{1}$. 


\section{Automorphismes Du Groupe De Cremona}

Soit $(x+b(y), y)$ dans $\varphi\left(\mathrm{T}_{2}\right) \cap \operatorname{ker} \pi$. Comme $\varphi\left(\mathrm{T}_{2}\right)$ est abélien, nous avons, pour tout $\nu$ dans $\pi\left(\varphi\left(\mathrm{T}_{2}\right)\right)$, l'égalité $b \circ \nu=b$. Le groupe $\mathrm{T}$ ne contenant pas d'élément de torsion, la proposition 3.3 et le lemme 4.6 assurent que $\pi(\varphi(\mathrm{T}))$, et donc $\pi\left(\varphi\left(\mathrm{T}_{2}\right)\right)$, est infini. Il s'en suit que $b$ est constant ; puisque $\varphi\left(\mathrm{T}_{2}\right)$ et $\mathrm{T}_{1}$ sont disjoints, $b$ est nul. Ainsi $\pi_{\mid \varphi\left(\mathrm{T}_{2}\right)}$ est injective et $\pi\left(\varphi\left(\mathrm{T}_{2}\right)\right)$ est non dénombrable. Le théorème 4.9 assure que $\varphi$ fixe $\mathrm{T}$ à conjugaison près ; par suite $\mathrm{T}_{2}$ est invariant $\operatorname{par} \varphi$.

\subsection{Groupes diagonaux}

Supposons maintenant que $\varphi\left(\mathrm{J}_{a}\right)=\mathrm{J}_{a}$ et $\varphi\left(\mathrm{T}_{i}\right)=\mathrm{T}_{i}$ pour $i=1,2$. Pour tout élément $(\alpha, \beta)$ de $\mathbb{C}^{*} \times \mathbb{C}^{*}$, notons $d_{\alpha, \beta}$ l'application $(\alpha x, \beta y)$. Introduisons les groupes suivants :

$$
\mathrm{D}_{1}=\left\{(a x, y) \mid a \in \mathbb{C}^{*}\right\} \quad \text { et } \quad \mathrm{D}_{2}=\left\{(x, a y) \mid a \in \mathbb{C}^{*}\right\} .
$$

Proposition 5.2. Soit $\varphi$ un automorphisme du groupe de Cremona. Supposons que $\varphi\left(\mathrm{J}_{a}\right)=\mathrm{J}_{a}$ et $\varphi\left(\mathrm{T}_{i}\right)=\mathrm{T}_{i}$ pour $i=1,2$. Alors, à conjugaison permise près, les groupes $\mathrm{D}_{i}$ sont fixés par $\varphi$.

Démonstration. D'après la proposition 5.1 , l'image $\varphi\left(\mathrm{D}_{2}\right)$ de $\mathrm{D}_{2}$ par $\varphi$ est un sous-groupe de $\{(x+$ $b(y), \nu(y)) \mid b \in \mathbb{C}(y), \nu \in \operatorname{PGL}(2, \mathbb{C})\}$. A conjugaison permise près nous pouvons supposer que $\varphi\left(t_{0,1}\right)=t_{0,1}$. Puisque $\mathrm{D}_{1}$ agit trivialement sur $\mathrm{T}_{2}$, et en particulier sur $t_{0,1}$, l'image de $\mathrm{D}_{1}$ par $\varphi$ est un sous-groupe de $\{(\varepsilon(x), y+c(x)) \mid \varepsilon \in \operatorname{PGL}(2, \mathbb{C}), c \in \mathbb{C}(x)\}$. Le groupe $\mathrm{D}_{2}$ agit par conjugaison sur $\mathrm{T}_{2}$ donc $\varphi\left(\mathrm{D}_{2}\right)$ est inclus dans :

$$
\left\{(x+a, b y+c) \mid a, c \in \mathbb{C}, b \in \mathbb{C}^{*}\right\} .
$$

Cette action est sans point fixe donc tout élément $(x+a, b y+c)$ de $\varphi\left(\mathrm{D}_{2}\right)$ satisfait l'alternative suivante : $c=0$ ou $b=1$. Si $b$ vaut 1 , alors $\varphi\left(\mathrm{D}_{2}\right) \cap \varphi(\mathrm{T})$ est non trivial ; par suite $c$ est nul et $\varphi\left(\mathrm{D}_{2}\right)$ est contenu dans $\left\{(x+a, b y) \mid a \in \mathbb{C}, b \in \mathbb{C}^{*}\right\}$. De même en considérant l'action de $\mathrm{D}_{1}$ sur $\mathrm{T}_{1}$ nous montrons que $\varphi\left(\mathrm{D}_{1}\right)$ est un sous-groupe de $\left\{(a x, y+b) \mid a \in \mathbb{C}^{*}, b \in \mathbb{C}\right\}$. Finalement comme $\varphi\left(\mathrm{D}_{1}\right)$ et $\varphi\left(\mathrm{D}_{2}\right)$ commutent, $\varphi$ préserve les groupes $\mathrm{D}_{i}$.

\subsection{Invariance point par point des groupes $\mathbf{T}_{\boldsymbol{i}}$ et $\mathbf{D}_{\boldsymbol{i}}$}

Rappelons le lemme 1.8 : soit $\varphi$ un automorphisme du groupe des transformations affines de la droite complexe. Alors $\varphi$ est la composée d'un automorphisme intérieur et de l'action d'un isomorphisme $d u$ corps $\mathbb{C}$. Cet énoncé nous permet d'établir un résultat de rigidité pour les groupes $\mathrm{T}$ et $\mathrm{D}$.

Lemme 5.3. Soit $\varphi$ un automorphisme du groupe de Cremona fixant les groupes $\mathrm{J}_{a}, \mathrm{~T}_{i}$ et $\mathrm{D}_{i}$. Quitte à conjuguer $\varphi$ par un automorphisme intérieur et un automorphisme du corps $\mathbb{C}$, les groupes $\mathrm{D}$ et T sont laissés invariants point par point.

Démonstration. Les groupes $\mathrm{T}_{i}$ et $\mathrm{D}_{i}$ étant fixes $\operatorname{par} \varphi$, il existe $\varphi_{1}$ et $\varphi_{2}$ deux automorphismes du groupe des transformations affines de la droite tels que, pour tout $(\alpha, \beta)$ dans $\mathbb{C}^{* 2}$ et pour tout $(r, s)$ dans $\mathbb{C}^{2}$, nous ayons :

$$
\varphi(\alpha x+r, \beta y+s)=\left(\varphi_{1}(\alpha x+r), \varphi_{2}(\beta y+s)\right)
$$

Le lemme 1.8 assure que :

$$
\varphi(\alpha x+r, \beta y+s)=\left(\tau_{1}(\alpha) x+\lambda \tau_{1}(r), \tau_{2}(\beta) y+\mu \tau_{2}(s)\right)
$$

où $\tau_{1}, \tau_{2}$ sont des automorphismes du corps $\mathbb{C}$ et $\lambda, \mu$ deux complexes non nuls.

Le groupe $\mathrm{J}_{a}$ étant invariant par $\varphi$, il existe $\xi$ dans $\mathbb{C}(y)$ tel que $\varphi(x+y, y)$ s'écrive $(x+\xi(y), y)$. Puisque, pour tout élément $\alpha$ de $\mathbb{C}^{*}$, l'élément $(x+y, y)$ commute à $d_{\alpha, \alpha}$, l'application $(x+\xi(y), y)$ commute à $d_{\tau_{1}(\alpha), \tau_{2}(\alpha)}$, autrement dit $\tau_{1}(\alpha) \xi\left(\tau_{2}(\alpha)^{-1} y\right)=\xi(y)$. Développons $\xi$ en série de Laurent à 


\section{J. DÉSERTI}

l'origine, $\xi(y)=\sum_{i \geqslant-N} \xi_{i} y^{i}$; l'égalité précédente conduit, pour tout $i \geqslant-N$, à $\left(\tau_{1}(\alpha) \tau_{2}(\alpha)^{-i}-1\right)$ $\xi_{i}=0$. Pour tout entier $i$ tel que $\xi_{i}$ soit non nul, $\tau_{1}(\alpha) \tau_{2}(\alpha)^{-i}$ vaut 1 . Comme $\tau_{1}$ est additif, l'égalité $\tau_{2}(\alpha)^{i}=\tau_{1}(\alpha)$ implique que $\tau_{2}^{i}$ est additif ; ceci n'est possible que si $i$ vaut 1 ce qui montre que $\xi$ est un monôme de degré un et que $\tau_{2}$ est égal à $\tau_{1}$. Ainsi $\varphi(\alpha x+r, \beta y+s)=$ $\left(\tau_{1}(\alpha) x+\lambda \tau_{1}(r), \tau_{1}(\beta) y+\mu \tau_{1}(s)\right)$. Quitte à conjuguer $\varphi$ par $d_{\tau_{1}(\lambda), \tau_{1}(\mu)}$, puis composer par l'action de l'automorphisme de corps $\tau_{1}$ nous obtenons $\varphi(\alpha x+r, \beta y+s)=(\alpha x+r, \beta y+s)$.

\subsection{Conclusion}

Lemme 5.4. Soit $\varphi$ un automorphisme du groupe de Cremona laissant les groupes $\mathrm{D}$ et $\mathrm{T}$ invariants point par point. Alors les involutions $(x, 1 / y),(y, x)$ et $\sigma$ sont fixées par $\varphi$.

Démonstration. Notons $\varsigma=\left(\varsigma_{1}, \varsigma_{2}\right)$ l'image de $( \pm y, \pm x)$ par $\varphi$. L'action de l'involution $( \pm y, \pm x)$ sur D implique que $\varsigma_{1}\left(d_{\alpha, \beta}\right)=\beta \varsigma_{1}(x, y)$ et $\varsigma_{2}\left(d_{\alpha, \beta}\right)=\alpha \varsigma_{2}(x, y)$ pour tous $\alpha$ et $\beta$ dans $\mathbb{C}^{*}$; ainsi $\varsigma_{1}$ et $\varsigma_{2}$ s'écrivent respectivement $\pm y$ et $\pm x$. La transformation $( \pm y, \pm x)$ agit par conjugaison sur $\mathrm{T}$ alors que $(-y, x)$ et $(y,-x)$ non, donc $\varsigma=( \pm y, \pm x)$. L'involution $(y, x)$ agit trivialement sur les éléments $t_{\alpha, \alpha}$ de $\mathrm{T}$ alors que $(-y,-x)$ non $;(y, x)$ et $(-y,-x)$ sont donc invariants par $\varphi$.

L'involution $(x, 1 / y)$ agit sur le groupe diagonal ; soit $d_{\alpha, \beta}$ un élément de $\mathrm{D}$, nous avons :

$$
(x, 1 / y) d_{\alpha, \beta}(x, 1 / y)=d_{\alpha, 1 / \beta} .
$$

En appliquant $\varphi$ nous obtenons, pour tous $\alpha, \beta$ dans $\mathbb{C}^{*}$ :

$$
\tau_{1}\left(d_{\alpha, \beta}\right)=\alpha \tau_{1}(x, y) \quad \text { et } \quad \tau_{2}\left(d_{\alpha, \beta}\right)=\tau_{2}(x, y) / \beta
$$

où $\tau=\left(\tau_{1}, \tau_{2}\right)$ désigne l'image de $(x, 1 / y)$ par $\varphi$. Par suite, $\tau(x, y)=( \pm x, \pm 1 / y)$. L'action de $(x, 1 / y)$ sur $t_{1,0}$ entraîne $: \varphi(x, 1 / y)=(x, \pm 1 / y)$.

L'égalité $((y, x)(x, 1 / y))^{2}=\sigma$ assure que $\varphi(\sigma)= \pm \sigma$ suivant que $(x, 1 / y)$ est envoyé sur luimême ou sur $(x,-1 / y)$. Notons $h=(x /(x-1),(x-y) /(x-1))$; comme l'a remarqué Gizatullin (voir [Giz99]), la transformation $(h \sigma)^{3}$ est triviale donc $(h \varphi(\sigma))^{3}$ doit aussi l'être. Nous en déduisons que l'involution de Cremona est invariante $\operatorname{par} \varphi$; par suite $(x, 1 / y)$ l'est aussi.

Démonstration du théorème 1.3. Soit $\varphi$ un automorphisme du groupe de Cremona. Le corollaire 4.3 permet de supposer que $J_{a}$ est invariant par $\varphi$. Nous montrons que si $\varphi\left(J_{a}\right)=J_{a}$, les groupes $T_{i}$ sont fixes $\operatorname{par} \varphi$ à conjugaison permise près (proposition 5.1) ; sous ces hypothèses nous obtenons, toujours à conjugaison permise près, que $\varphi\left(\mathrm{D}_{i}\right)=\mathrm{D}_{i}$ pour $i=1,2$ (proposition 5.2). Ainsi, à automorphisme de corps et conjugaison près, les groupes $\mathrm{T}_{i}$ et $\mathrm{D}_{i}$ sont invariants point par point (lemme 5.3) ; il s'en suit que les involutions $(y, x),(x, 1 / y)$ et $\sigma$ sont envoyées sur elles-mêmes par $\varphi$ (lemme 5.4). Or le groupe engendré par le groupe diagonal, le groupe des translations, $(y, x)$ et $(x, 1 / y)$ contient $\mathrm{PGL}(3, \mathbb{C})$ donc le théorème 1.2 permet de conclure.

\section{Compléments}

Soit $H$ un sous-groupe de $\operatorname{Bir}\left(\mathbb{P}^{2}(\mathbb{C})\right)$ de la forme $(\mathbb{Z} / p \mathbb{Z})^{r}$ avec $p$ premier ; d'après $[\operatorname{Kan} 95$, Wim96, Bea05], si $p \geqslant 5$, alors $r \leqslant 2$. Notons que $\operatorname{Aut}\left(\mathbb{P}^{1}(\mathbb{C})\right)=\operatorname{Bir}\left(\mathbb{P}^{1}(\mathbb{C})\right)$ ne contient pas $(\mathbb{Z} / p \mathbb{Z})^{n}, n>1$, $p>2 ; \operatorname{comme} \operatorname{Aut}\left(\mathbb{P}^{n}(\mathbb{C})\right)$, et donc $\operatorname{Bir}\left(\mathbb{P}^{n}(\mathbb{C})\right)$, contient $(\mathbb{Z} / p \mathbb{Z})^{n}$ dès que $n \geqslant 2$, nous en déduisons la remarque suivante.

Remarque 6.1. Les groupes $\operatorname{Bir}\left(\mathbb{P}^{2}(\mathbb{C})\right)$ et $\operatorname{Bir}\left(\mathbb{P}^{n}(\mathbb{C})\right)$ sont isomorphes si et seulement si $n=2$.

Nous pouvons déduire du théorème 1.3 un résultat sur le groupe d'automorphismes du semigroupe des transformations rationnelles de $\mathbb{P}^{2}(\mathbb{C})$ (la preuve est quasiment identique à celle du corollaire 6.4, [Dés06]) ; ce semi-groupe contient $\operatorname{Bir}\left(\mathbb{P}^{2}(\mathbb{C})\right)$ nous pouvons donc parler d'automorphisme intérieur. 


\section{Automorphismes Du Groupe De Cremona}

Corollaire 6.2. Un isomorphisme du semi-groupe des transformations rationnelles de $\mathbb{P}^{2}(\mathbb{C})$ dans lui-même est intérieur à composition près par un automorphisme du corps $\mathbb{C}$.

Nous allons démontrer le corollaire 1.4 dont nous rappelons l'énoncé :

Soient $S$ une surface projective complexe et $\varphi$ un isomorphisme entre $\operatorname{Bir}(S)$ et $\operatorname{Bir}\left(\mathbb{P}^{2}(\mathbb{C})\right)$. Il existe une transformation birationnelle $\psi: S \rightarrow \mathbb{P}^{2}(\mathbb{C})$ et un automorphisme $\tau$ du corps $\mathbb{C}$ tels que, pour tout $f$ dans $\operatorname{Bir}(S)$, nous ayons : $\varphi(f)=\tau\left(\psi f \psi^{-1}\right)$.

Démonstration du corollaire 1.4. Si la dimension de Kodaira de $S$, que nous notons $\operatorname{kod}(S)$, est deux, alors tout élément de $\operatorname{Bir}(S)$ est d'ordre fini. Lorsque $\operatorname{kod}(S)$ vaut un, $\operatorname{Bir}(S)$ préserve une fibration elliptique et est virtuellement abélien (voir [BHPV04]). Dans chacun de ces cas, nous constatons que $\operatorname{Bir}(S)$ et $\operatorname{Bir}\left(\mathbb{P}^{2}(\mathbb{C})\right)$ ne sont pas isomorphes.

Supposons que $\operatorname{kod}(S)$ soit nulle. $\operatorname{Si} \operatorname{Bir}(S)$ et $\operatorname{Bir}\left(\mathbb{P}^{2}(\mathbb{C})\right)$ sont isomorphes, alors ce dernier contient un sous-groupe distingué abélien d'indice dénombrable $H$ (voir [BHPV04]). Considérons l'inclusion $j$ de $\operatorname{PGL}(3, \mathbb{C})$ dans $\operatorname{Bir}\left(\mathbb{P}^{2}(\mathbb{C})\right)$ et la projection $p$ de $\operatorname{Bir}\left(\mathbb{P}^{2}(\mathbb{C})\right)$ sur $\operatorname{Bir}\left(\mathbb{P}^{2}(\mathbb{C})\right) / H$. Puisque $\operatorname{PGL}(3, \mathbb{C})$ est simple et que $\operatorname{Bir}\left(\mathbb{P}^{2}(\mathbb{C})\right) / H$ est dénombrable, $\operatorname{ker}(p \circ j)=\operatorname{PGL}(3, \mathbb{C})$ est contenu dans $H$ : impossible.

Si $\operatorname{kod}(S)$ est $-\infty$, alors $S$ est une surface réglée non rationnelle ou une surface rationnelle. Plaçons nous dans la première éventualité ; il existe une fibration rationnelle $P: S \rightarrow B$ localement triviale. Cette fibration est préservée $\operatorname{par} \operatorname{Bir}(S)$, i.e. il existe $\rho: \operatorname{Bir}(S) \rightarrow \operatorname{Aut}(B)$ tel que, pour tout élément $f$ de $\operatorname{Bir}(S)$, nous ayons $P \circ f=\rho(f) \circ P$. Supposons que $\operatorname{Bir}(S)$ et $\operatorname{Bir}\left(\mathbb{P}^{2}(\mathbb{C})\right)$ soient isomorphes, alors $\mathrm{SL}(3, \mathbb{C})$ s'injecte via $j$ dans $\operatorname{Bir}(S)$; par simplicité, $\operatorname{SL}(3, \mathbb{C})$ s'injecte dans ker $\rho=\operatorname{PGL}(2, \mathrm{k})$, où $\mathrm{k}$ désigne le corps des fonctions méromorphes de $B$, ce qui est impossible. La surface $S$ est donc nécessairement rationnelle.

Corollaire 6.3. Le groupe des automorphismes du corps $\mathbb{C}(x, y)$ est isomorphe au groupe des automorphismes du groupe de Cremona.

Démonstration. Soit $\varphi$ un automorphisme du corps $\mathbb{C}(x, y)$. Les constantes sont les seuls éléments de $\mathbb{C}(x, y)$ à posséder une racine $n$-ième pour tout $n$. Il s'en suit que $\mathbb{C}$ est invariant par $\varphi$; par suite, à automorphisme de corps près, $\varphi_{\mid \mathbb{C}}$ est trivial. Notons $f$ la transformation birationnelle définie par $f=(\varphi(x), \varphi(y))$; pour tout couple $(i, j)$ d'entiers, nous avons $\varphi\left(x^{i} y^{j}\right)=x^{i} y^{j} \circ f$. Ceci conduit à $\varphi(P)=P \circ f$ pour tout polynôme $P$; nous en déduisons une égalité du même type pour toute fonction rationnelle $R$. Ainsi tout automorphisme de $\mathbb{C}(x, y)$ s'obtient par la composition de l'action d'un automorphisme du corps $\mathbb{C}$ et d'une transformation birationnelle.

\section{REMERCIEMENTS}

Merci à S. Cantat et D. Cerveau pour leur enthousiasme et pour les nombreuses discussions que nous avons eues. Je remercie S. Lamy et le rapporteur pour leurs remarques et suggestions.

\section{BIBLIOGRAPHIE}

Alb02 M. Alberich-Carramiñana, Geometry of the plane Cremona maps, Lecture Notes in Mathematics, vol. 1769 (Springer, Berlin, 2002).

Ban86 A. Banyaga, On isomorphic classical diffeomorphism groups. I, Proc. Amer. Math. Soc. 98 (1986), $113-118$.

Ban97 A. Banyaga, The structure of classical diffeomorphism groups, Mathematics and its Applications, vol. 400 (Kluwer, Dordrecht, 1997).

Bea05 A. Beauville, p-elementary subgroups of the Cremona group, Preprint (2005), arXiv:math.AG/0502123. 


\section{Automorphismes Du groupe De Cremona}

BHPV04 W. P. Barth, K. Hulek, C. A. M. Peters and A. Van de Ven, Compact complex surfaces, second edition, Ergebnisse der Mathematik und ihrer Grenzgebiete (3), vol. 4 (Springer, Berlin, 2004).

Can99 S. Cantat, Dynamique des automorphismes des surfaces complexes compactes, Thèse, École Normale Supérieure de Lyon (1999).

Cas01 G. Castelnuovo, Le trasformationi generatrici del gruppo cremoniano nel piano, Atti R. Accad. Sci. Torino 36 (1901), 861-874.

CF03 S. Cantat and C. Favre, Symétries birationnelles des surfaces feuilletées, J. reine angew. Math. $\mathbf{5 6 1}$ (2003), 199-235.

Dés06 J. Déserti, Sur le groupe des automorphismes polynomiaux du plan affine, J. Algebra 297 (2006), 584-599.

Die55 J. Dieudonné, La géométrie des groupes classiques, Ergebnisse der Mathematik und ihrer Grenzgebiete (NF), vol. 5 (Springer, Berlin, 1955).

Enr93 F. Enriques, Sui gruppi continui di transformazioni cremoniane nel piano, Rend. Accad. Lincei 2 (1893), 468-473.

Fil82 R. P. Filipkiewicz, Isomorphisms between diffeomorphism groups, Ergodic Theory Dynam. Systems 2 (1982), 159-171.

Giz99 M. Gizatullin, On some tensor representations of the Cremona group of the projective plane, in New trends in algebraic geometry, Warwick, 1996, London Mathematical Society Lecture Note Series, vol. 264 (Cambridge University Press, Cambridge, 1999), 111-150.

Jun42 H. W. E. Jung, Über ganze birationale Transformationen der Ebene, J. reine angew. Math. 184 (1942), 161-174.

Kan95 S. Kantor, Theorie der endlichen Gruppen von eindeutigen Transformationen in der Ebene (Mayer \& Mullen, Berlin, 1895).

Lam01 S. Lamy, L'alternative de Tits pour Aut[ $\left.\mathbb{C}^{2}\right]$, J. Algebra 239 (2001), 413-437.

Lam02 S. Lamy, Une preuve géométrique du théorème de Jung, Enseign. Math. (2) 48 (2002), 291-315.

MZ55 D. Montgomery and L. Zippin, Topological transformation groups (Interscience, New York, 1955).

Tak79 F. Takens, Characterization of a differentiable structure by its group of diffeomorphisms, Bol. Soc. Brasil. Mat. (NS) 10 (1979), 17-25.

Ume82 H. Umemura, On the maximal connected algebraic subgroups of the Cremona group. I, Nagoya Math. J. 88 (1982), 213-246.

Whi63 J. V. Whittaker, On isomorphic groups and homeomorphic spaces, Ann. of Math. (2) 78 (1963), 74-91.

Wim96 A. Wiman, Zur Theorie der endlichen Gruppen von birationalen Transformationen in der Ebene, Math. Ann. 48 (1896), 195-240.

Julie Déserti julie.deserti@univ-rennes1.fr

IRMAR, UMR 6625 du CNRS, Université de Rennes I, 35042 Rennes, France 